\title{
ENGINEERING AND ECONOMIC APPLICATIONS OF COMPLEMENTARITY PROBLEMS*
}

\author{
M. C. FERRIS ${ }^{\dagger}$ AND J. S. PANG ${ }^{\ddagger}$
}

\begin{abstract}
This paper gives an extensive documentation of applications of finite-dimensional nonlinear complementarity problems in engineering and equilibrium modeling. For most applications, we describe the problem briefly, state the defining equations of the model, and give functional expressions for the complementarity formulations. The goal of this documentation is threefold: (i) to summarize the essential applications of the nonlinear complementarity problem known to date, (ii) to provide a basis for the continued research on the nonlinear complementarity problem, and (iii) to supply a broad collection of realistic complementarity problems for use in algorithmic experimentation and other studies.
\end{abstract}

Key words. complementarity problems, variational inequalities, frictional contact, structural engineering, economic equilibria, transportation planning, Nash equilibria

AMS subject classifications. 90C33, 90C50, 70E15, 73K05, 90A14, 90D10

PII. S0036144595285963

1. Introduction. As a result of more than three decades of research, the subject of complementarity problems, with its diverse applications in engineering, economics, and sciences, has become a well-established and fruitful discipline within mathematical programming. Several monographs $[34,79,135]$ and surveys $[72,148]$ have documented the basic theory, algorithms, and applications of complementarity problems and their role in optimization theory.

An important reason why complementarity problems are so pervasive in engineering and economics is because the concept of complementarity is synonymous with the notion of system equilibrium. The balance of supply and demand is central to all economic systems; mathematically, this fundamental equation in economics is often described by a complementary relation between two sets of decision variables. For instance, the classical Walrasian law of competitive equilibria of exchange economies [203] can be formulated as a nonlinear complementarity problem in the price and excess demand variables. The complementarity condition expresses the fact that the excess demand of a commodity must be zero if its price is positive; similarly, the price of the commodity must be zero if there is positive excess supply. Complementarity is also central to all constrained optimization problems. The well-known complementary slackness property in linear programming exemplifies the fundamental role of complementarity in optimization; this property persists in nonlinear programs and variational inequalities. Optimization is a recurring theme in numerous engineering applications; however, many engineering systems involve the notion of equilibrium without an objective being optimized. For instance, the renowned Wardrop principle

*Received by the editors May 12, 1995; accepted for publication (in revised form) January 14, 1997.

http://www.siam.org/journals/sirev/39-4/28596.html

${ }^{\dagger}$ Computer Sciences Department, University of Wisconsin, Madison, WI 53706 (ferris@cs. wisc.edu). The work of this author was based on research supported by National Science Foundation grant CCR-9157632, Department of Energy grant DE-FG03-94ER61915, and Air Force Office of Scientific Research grant F49620-94-1-0036.

${ }_{\ddagger}^{\ddagger}$ Department of Mathematical Sciences, The Johns Hopkins University, Baltimore, MD 212182689 (jsp@vicp.mts.jhu.edu). The work of this author was based on research supported by National Science Foundation grant CCR-9213739 and by Office of Naval Research grant N00014-93-1-0228. 
of user equilibrium in traffic theory [205] has a natural formulation as a nonlinear complementarity system; in this case, the complementarity condition is a behavioral statement about the users of a traffic network who are postulated to take shortest paths in the network. Another example is the physical contact of mechanical structures; here complementarity is between the contact force and the gap (i.e., the distance) between the bodies in contact: the contact force is positive only if there is contact (that is, if the gap is zero). A major objective of this paper is to elucidate the pervasiveness of complementarity in these and other important engineering and economic applications.

Among the many facets of research in complementarity problems, one that has received wide attention in recent years is the development of robust and efficient algorithms for solving the ever increasing applications of these problems. There is presently a wide variety of computational methods for solving complementarity problems. Unlike the fixed-point homotopy methods $[63,197]$ some of which are known to be practically deficient for solving realistic equilibrium applications of the complementarity problem (see [72] and the references therein) most of the contemporary computational schemes are designed with the goal of removing these deficiencies and meeting the need of solving large-scale applications efficiently. These computational methods include the following:

- extensions of Newton's method for nonlinear equations [73, 84, 129, 147, 163] that replace the direction finding routines with complementarity problems,

- a path search method $[41,42,162]$ that uses a generalization of a line search technique,

- quadratic programming-based algorithms $[15,59,60,149]$ that derive extensions of the Gauss-Newton methodology,

- differentiable optimization based descent methods [55, 88, 123, 125, 199] that reformulate the complementarity relationships as a nonlinear equation or program,

- projection and proximal methods $[8,9,48,132,182]$ that extend projected gradient methods,

- smoothing techniques $[22,21,23,24,89,58,160]$ that replace the nonsmooth equations with differentiable approximations,

- and interior point methods $[5,14,22,21,66,81,99,100,101,102,131,180$, 204, 207] based on removing inequalities by an interior penalty.

Along with the research in the design and analysis of algorithms comes the recognition that the linkage of these algorithms with such mathematical programming modeling languages as GAMS [19] and AMPL [51] is extremely important for the algorithms to become easily accessible to practitioners and academic researchers. Motivated by the desire to solve complex economic equilibrium problems, Rutherford [172] developed a modeling system for applied general equilibrium systems and subsequently $[174,175]$ extended the original GAMS modeling language to enable the treatment of complementarity constraints. Further work on modeling language interfaces of complementarity algorithms can be found in the Ph.D. thesis of Dirkse [39] and the paper [43].

Numerical experimentation has always been an important part of algorithmic development. The paper [73] is perhaps the first to report extensive computational results with an algorithm for solving realistic nonlinear complementarity problems arising from various economic applications. This set of test problems has since been augmented in $[59,105,149,208]$; a model library containing a summary of these 
problems is formulated in the GAMS [40] and AMPL languages [39, Chapter 3]. Computational results comparing several algorithms on these test problems can be found in [13].

Fueled by a desire to expand such a model library and to provide a basis for the continued research on complementarity problems, we decided to undertake the daunting task of uncovering all interesting applications of these problems known to us. The result of our effort is a large collection of realistic complementarity problems of various type, size, complexity, and computational difficulty. Collectively, these problems are expected to pose new challenges for a general researcher in the field and particularly for an algorithm designer.

The rest of this paper is divided into three major sections. The next section sets up the notation used in the paper and describes the various types of complementarity problems that appear later. Section 3 describes the engineering problems and gives their complementarity formulations. Section 4 does the same for the economic equilibrium problems. Finally we give some concluding remarks in the fifth and last section.

2. Types of complementarity problems. Complementarity problems come in different types. In addition to the familiar ones - linear, nonlinear, generalizedsuch adjectives as "mixed, horizontal, vertical, extended" have been coined to describe a complementarity problem of a particular type. In this section we give an overview of the various problems that we consider collectively as complementarity problems. Many of the applications that we detail in the sequel have naturally occurring complementarity forms, and in our description of the problems we will maintain this natural form.

2.1. Nonlinear complementarity problems. This classical problem, defined by a nonlinear function $F: \mathbf{R}^{n} \rightarrow \mathbf{R}^{n}$, is to find an $x \in \mathbf{R}^{n}$ such that

$$
\operatorname{NCP}(F): \quad 0 \leq x \perp F(x) \geq 0,
$$

where we use the perp notation " $\perp$ " to signify that in addition to the stated inequalities, the equation $x^{T} F(x)=0$ also holds. Note that since $x^{T} F(x)=\sum_{i=1}^{n} x_{i} F_{i}(x)$, this can be equivalently stated as

$$
x \geq 0, \quad F(x) \geq 0, \quad x_{i} F_{i}(x)=0, \quad i=1,2, \ldots, n .
$$

In effect then complementarity states that either $x_{i}$ or $F_{i}(x)$ must be zero for each $i=1,2, \ldots, n$. It is easy to see that $\operatorname{NCP}(F)$ is equivalent to the following problem of solving the nonsmooth equation

$$
\min (x, F(x))=0,
$$

where the min operation is taken componentwise. Complementarity problems of this form arise as the Karush-Kuhn-Tucker (KKT) conditions of a constrained nonlinear program

$$
\begin{array}{ll}
\operatorname{minimize} & \theta(x) \\
\text { subject to } & g(x) \leq 0, \quad x \geq 0,
\end{array}
$$

where $\theta: \mathbf{R}^{n} \rightarrow \mathbf{R}$ is a continuously differentiable real-valued function and $g: \mathbf{R}^{n} \rightarrow$ $\mathbf{R}^{m}$ is a continuously differentiable vector-valued function. 
2.2. Variational inequalities. In practice, many problems have lower and/or upper bounds on the variables, instead of the standard nonnegativity shown above. Most generally, a problem may have both lower and upper bounds on some variables, only lower or upper bounds on other variables, and no such bounds at all on the remaining variables. To accommodate such a general problem, the following variational inequality format has been frequently used in the literature:

VI $(F,[l, u]): \quad \quad$ find $x \in[\ell, u]$ such that $(y-x)^{T} F(x) \geq 0 \quad \forall y \in[\ell, u]$,

where $\ell$ and $u$ are $n$-dimensional vectors with $\ell_{i} \in[-\infty, \infty)$ and $u_{i} \in\left(\ell_{i}, \infty\right]$, and

$$
[\ell, u] \equiv\left\{x \in \mathbf{R}^{n}: \ell \leq x \leq u\right\} .
$$

If $\ell_{i}=0, u_{i}=\infty$ for each $i=1,2, \ldots, n$, it follows easily that VI $(F,[0, \infty])$ is precisely $\operatorname{NCP}(F)$. In several places the $\mathrm{VI}(F,[\ell, u])$ is termed a box-constrained or a rectangular variational inequality, the box referring to the set $[\ell, u]$.

In many applications, some of the underlying conditions are defined by a system of nonlinear equations, while the complementarity conditions are only applied to some of the variables and functions. This leads to a type of problem generally termed a mixed nonlinear complementarity problem and can be written in the following form:

$$
\begin{gathered}
F_{\mathcal{I}}(x)=0, \quad x_{\mathcal{I}} \text { free, } \\
0 \leq x_{\mathcal{J}} \perp F_{\mathcal{J}}(x) \geq 0,
\end{gathered}
$$

where $\mathcal{I}$ and $\mathcal{J}$ form a partition of $\{1,2, \ldots, n\}$. To incorporate a free variable $x_{i}$ into a model, we would set $\ell_{i}=-\infty$ and $u_{i}=\infty$. The mixed nonlinear complementarity problem can be recovered as a special case of VI $(F,[\ell, u])$ by setting $\ell_{i}=-\infty$, $u_{i}=\infty$ for $i \in \mathcal{I}$, and $\ell_{i}=0, u_{i}=\infty$ for $i \in \mathcal{J}$. Due to this fact, the box-constrained variational inequality is typically termed a mixed complementarity problem. Notice that the bounds $\ell$ and $u$ on the variables implicitly define the constraints associated with the function $F$.

A special case of a mixed complementarity problem is where $\mathcal{I}=\{1,2, \ldots, n\}$, resulting in a system of nonlinear equations:

NE :

$$
F(x)=0 .
$$

The VI $(F,[l, u] \cap X)$, where $X=\{x: A x=b\}$ and $A$ is an $m \times n$ matrix, is also easily transformed into a box-constrained variational inequality by introducing multipliers for the linear equality constraints. It is easy to show that VI $(F,[l, u] \cap X)$ is equivalent to $\mathrm{VI}\left(H,[l, u] \times \mathbf{R}^{m}\right)$, where

$$
H(x, \lambda)=\left[\begin{array}{c}
F(x)+A^{T} \lambda \\
-A x+b
\end{array}\right] .
$$

More generally, if $X=\{x: g(x) \leq 0, h(x)=0\}$, where $g: \mathbf{R}^{n} \rightarrow \mathbf{R}^{m}$ and $h:$ $\mathbf{R}^{n} \rightarrow \mathbf{R}^{s}$ are continuously differentiable functions, then under a suitable constraint qualification, for any solution $x$ of the VI $(F,[l, u] \cap X)$, there must exist multipliers $\lambda \in \mathbf{R}^{m}$ and $\eta \in \mathbf{R}^{s}$ such that the triple $(x, \lambda, \eta)$ is a solution of the VI $(H,[l, u] \times$ $\left.[0, \infty) \times \mathbf{R}^{s}\right)$, where

$$
H(x, \lambda, \mu)=\left[\begin{array}{c}
L(x, \lambda, \mu) \\
-g(x) \\
h(x)
\end{array}\right]
$$


with

$$
L(x, \lambda, \mu) \equiv F(x)+\sum_{i=1}^{m} \lambda_{i} \nabla g_{i}(x)+\sum_{j=1}^{s} \mu_{j} \nabla h_{j}(x)
$$

is the vector-valued Lagrangian function for the $\mathrm{VI}(F,[l, u] \times X)$.

2.3. Vertical complementarity problems. There is a certain lack of symmetry in the nonlinear complementarity problem as can be seen in the formulation $\min (x, F(x))=0$. One of the functions in this formulation is quite arbitrary while the other is the identity. Many commonly occurring problems actually have a more general form

$$
\min \left(F^{1}(x), F^{2}(x)\right)=0
$$

for two given functions $F^{1}, F^{2}: \mathbf{R}^{n} \rightarrow \mathbf{R}^{n}$. Of course, it is possible to have more than two functions in the above equation; the resulting problem is called the vertical nonlinear complementarity problem:

$\operatorname{VCP}(F): \quad \min \left(F^{1}(x), F^{2}(x), \ldots, F^{m}(x)\right)=0$.

Clearly, this means that $F_{i}^{j}(x) \geq 0$ for all $i=1,2, \ldots, n$ and $j=1,2, \ldots, m$ and for each component $i, F_{i}^{j}(x)=0$ for at least one $j$. The affine version of this problem (that is when all the functions $F^{i}$ are affine) was introduced by Cottle and Dantzig [31]; it has been treated in several studies [44, 65, 80, 124].

The VCP can be equivalently cast as a box-constrained variational inequality (more precisely, a mixed nonlinear complementarity problem) by introducing extra variables $z^{j} \in \mathbf{R}^{n}, j=2,3, \ldots, m$; the equivalent formulation is

$$
\begin{gathered}
\sum_{k=2}^{m} z^{k}=F^{1}(x), \\
0=\min \left(z^{j}, F^{j}(x)-\sum_{k=j+1}^{m} z^{k}\right), \quad j=2, \ldots, m-1, \\
0=\min \left(z^{m}, F^{m}(x)\right) .
\end{gathered}
$$

It can be seen that if $x$ solves the $\operatorname{VCP}(F)$, then the above equations are satisfied with

$$
\begin{aligned}
& z^{j} \equiv \min \left(F^{1}(x), \ldots, F^{j-1}(x)\right)-\sum_{k=j+1}^{m} z^{k}, \quad j=2, \ldots, m-1, \\
& z^{m} \equiv \min \left(F^{1}(x), \ldots, F^{m-1}(x)\right)
\end{aligned}
$$

conversely, if (2.3) holds, then $x$ solves the $\operatorname{VCP}(F)$. (Danny Ralph at the University of Melbourne had previously communicated to the authors a related formulation of the $\mathrm{VCP}$ as a normal equation.)

Notice that the number of variables in problem (2.3) is $m n$, compared with just $n$ in the original min formulation of the $\operatorname{VCP}(F)$. Some algorithms for solving the VCP (e.g., the Gauss-Newton method for nonsmooth equations in [150]) may exploit the particular structure of the min formulation much more effectively than treating the variational inequality directly. However, many of the variables introduced in (2.3) arise in a purely linear fashion, so a general purpose solver for the VI may be able to exploit this fact. 
2.4. Saddle-point problems. These problems are generalizations of nonlinear programs and provide a rich source of complementarity problems and variational inequalities. In general, given two nonempty sets $X \subseteq \mathbf{R}^{n}$ and $Y \subseteq \mathbf{R}^{m}$ and a function $\mathcal{L}: \mathbf{R}^{n} \times \mathbf{R}^{m} \rightarrow \mathbf{R}$, the saddle-point problem associated with the triple $(X, Y, \mathcal{L})$ is to find a pair of vectors $\left(x^{*}, y^{*}\right) \in X \times Y$, called a saddle point, such that

$$
\mathcal{L}\left(x^{*}, y\right) \leq \mathcal{L}\left(x^{*}, y^{*}\right) \leq \mathcal{L}\left(x, y^{*}\right) \quad \text { for all }(x, y) \in X \times Y .
$$

Suppose that $\mathcal{L}$ is a continuously differentiable convex-concave function (convexityconcavity means that $\mathcal{L}(x, y)$ is a convex function in $x$ for each fixed $y$ and a concave function in $y$ for each fixed $x)$, and also that $X$ and $Y$ are convex sets; then $\left(x^{*}, y^{*}\right)$ is a saddle point if and only if it solves the VI $(F, K)$, where

$$
K=X \times Y \quad \text { and } \quad F(x, y)=\left(\begin{array}{c}
\nabla_{x} \mathcal{L}(x, y) \\
-\nabla_{y} \mathcal{L}(x, y)
\end{array}\right) .
$$

The proof of this equivalence is elementary; see [164, section 36] for more discussion of these saddle-point problems.

In a series of recent papers $[165,167,168,169]$, an important special case of the above saddle-point problem was introduced as a modeling tool for various stochastic programming, multistage optimization, and optimal control problems. In this case, $X$ and $Y$ are both polyhedral sets and $\mathcal{L}$ is a linear-quadratic function:

$$
\mathcal{L}(x, y)=p^{T} x+\frac{1}{2} x^{T} P x-x^{T} R y+q^{T} y-\frac{1}{2} y^{T} Q y
$$

for some matrices $P, Q$, and $R$ and vectors $p$ and $q$ of appropriate dimensions. This special saddle-point problem leads to an affine VI $(F, K)$ in which the defining set $K$ is polyhedral and the function $F$ is affine; the name "extended linear-quadratic program" (ELQP) was used by the authors of the cited papers to signify the connection of this problem with standard linear and quadratic programming in classical optimization theory. Special computational schemes for solving the ELQP were developed in [45, $166,210,211]$.

All of the problems that we give in sections 3 and 4 will have one of the above forms. We have shown that all these forms can be reduced to VI $(F,[\ell, u])$ for particular choices of $\ell$ and $u$ and perhaps with the addition of extra variables and constraints. This paper does not address the issue of what form will be the best for any particular algorithm. This area of research is the subject of a separate investigation.

2.5. Mathematical programs with equilibrium constraints. Complementarity problems and, more generally, variational inequalities, often appear as constraints of an optimization problem. In [71], the term "mathematical programs with equilibrium constraints" or, simply, MPECs, was coined for this class of constrained optimization problems. In what follows, we will state the general formulation of the MPEC and refer the reader to the recent monograph [117] for a comprehensive study of this problem, including some historical accounts, an extensive theory, and several iterative algorithms for computing stationary points.

In general, an MPEC consists of two sets of variables: the "first-level" variable $x \in$ $\mathbf{R}^{n}$ and the "second-level" variable $y \in \mathbf{R}^{m}$. In a typical application, $x$ contains the design variables whereas $y$ contains the primary variables of a variational inequality that is parametrized by $x$. The following are given: the first-level objective function $f: \mathbf{R}^{n+m} \rightarrow \Re$, the joint feasible region $Z \subseteq \mathbf{R}^{n+m}$, the second-level "equilibrium 
function" $F: \mathbf{R}^{n+m} \rightarrow \Re^{m}$, and a set-valued mapping $C: \mathbf{R}^{n+m} \rightarrow \mathbf{R}^{m}$ where for each $x \in \mathbf{R}^{n}, C(x) \subseteq \mathbf{R}^{m}$ is the (possibly empty) constraint set of the second-level VI. The MPEC is

$$
\begin{array}{ll}
\operatorname{minimize} & f(x, y) \\
\text { subject to } & (x, y) \in Z \\
\text { and } & y \text { solves VI }(F(x, \cdot), C(x)) .
\end{array}
$$

In the important case where the VI $(F(x, \cdot), C(x))$ is equivalent to a complementarity system, the above MPEC can be formulated equivalently as a minimization problem with mixed complementarity constraint:

$$
\begin{array}{ll}
\operatorname{minimize} & \tilde{f}(x, u, v, w) \\
\text { subject to } & (x, u, v, w) \in \tilde{Z}, \\
& H(x, u, v, w)=0, \\
\text { and } & 0 \leq u \perp v \geq 0,
\end{array}
$$

where $(u, v, w)$ is the concatenation of the second-level variables, namely, $y$, the slacks and multipliers of the constraints that define the set $C(x), \tilde{f}$ and $\tilde{Z}$ are, respectively, the function $f$ and set $Z$ redefined in terms of the variables $(x, u, v, w)$, and $H$ is a vector-valued function that summarizes the functional constraints of the second-level VI.

The MPEC has many important applications in the areas of engineering design and economic planning. Indeed many of the problems described in the next two sections contain data that are design variables of an MPEC; these applications are therefore the second-level problems of the MPECs. An example is the Stackelberg game problem [184] which can be formulated as an MPEC with the second-level VI being a Nash game (see subsection 4.3). Since a full discussion of the diverse applications of the MPEC is beyond the scope of this paper, subsequently, we will mention a couple of these applications. We refer the interested reader to the monograph [117] for an extensive treatment of the MPEC.

3. Engineering applications. We divide the engineering problems discussed in this section into several categories: contact mechanics problems, structural mechanics problem, nonlinear obstacle problems, elastohydrodynamic lubrication problems, and traffic equilibrium problems. We also include a discrete-time optimal control application that can be formulated as an extended linear-quadratic programming problem. For each of these classes of problems, there is a substantial amount of literature, and other solution approaches have been suggested. The focus of our discussion is on the complementarity approach to these problems. In each case, we will give some background and a brief description of the physical problem, explain the defining equations, and present the complementarity formulations along with their functional expressions.

Since we are not experts in the disciplines of the problems described herein, our classification of the problems is perhaps not the most appropriate, especially in view of the fact that several of them are based on related physical and mechanical principles. Also, in describing the problems we have relied heavily on selected references which are most comprehensible to us, as well as on our own work pertaining to these applications.

3.1. Contact mechanics problems. Physical contacts between two or more solid bodies is a pervasive phenomenon in mechanics. According to [94], the treatment 
of frictionless contact problems by a mathematical programming approach dates back to $[30,52]$. Solution methods for such problems were based on quadratic programming formulations. Recent references on these problems include [3, 16, 17, 91, 92]. Contact problems with Coulomb friction have been treated extensively by Klarbring and his colleagues $[18,82,90,93,94,95,96,97]$ as well as in $[4,108,109,111,115$, $116,145,187,191]$. Mathematical programming techniques used for solving the latter problems include linear complementarity methods, sequential quadratic programming algorithms, Newton methods for generalized equations, and B-differentiable equations. One recent area of applications of frictional contact problems occurs in robotics research; see [11, 12, 151, 153, 161, 177, 198] and [145, section 14.1].

In their general form, frictional contact problems are modeled in terms of some abstract mappings describing the contact surfaces and body motions; their formulations are based on continuum mechanics principles. For computational purposes, these continuum formulations are often discretized by finite-element methods and in the case of time-dependent problems, by a stepwise incremental procedure which divides the time duration into several intervals. Such discretizations result in some finite-dimensional problems solvable by numerical methods. In what follows, we describe two contact problems in a finite-dimensional setting, one in the context of robot task planning and the other pertaining to contact between two elastic bodies.

3.1.1. A dynamic rigid-body model. In the three-dimensional rigid-body frictional contact model described in [151, 198], a rigid object comes into contact with a number of manipulator links at a finite number of points, and the contact forces obey a Coulomb friction law. At the instant the model is formulated (i.e., the current time), there are $n_{c}$ contact points which are classified as rolling contacts or sliding contacts. By definition, the normal components of the relative velocity at all contact points are zero. A contact point $i$ is rolling if the tangential and orthogonal components of velocity at that point are also zero; a contact point $i$ is sliding if either the tangential component $v_{i t}$ or the orthogonal component $v_{i o}$ of the velocity (which are given) is nonzero. Let $\mathcal{R}$ and $\mathcal{S}$ be two partitioning subsets of $\left\{1, \ldots, n_{c}\right\}$ denoting, respectively, the rolling and sliding contacts. Given a set of external forces (gravitational, centripetal, joint torques), the model calls for the determination of the linear accelerations $\left\{a_{i n}, a_{i t}, a_{i o}\right\}$ and the contact forces $\left\{c_{i n}, c_{i t}, c_{i o}\right\}$ at all contact points $i=1, \ldots, n_{c}$, where the subscripts $n, t, o$ denote the three components (normal, tangential, orthogonal) of the accelerations and forces, so that certain kinematic acceleration constraints, the Signorini nonpenetration condition, and Coulomb's friction law are satisfied. Specifically, letting

$$
\begin{aligned}
& a_{n} \equiv\left(a_{i n}\right)_{i=1}^{n_{c}}, \quad a_{t}=\left(a_{i t}\right)_{i=1}^{n_{c}}, \quad a_{o} \equiv\left(a_{i o}\right)_{i=1}^{n_{c}}, \\
& c_{n} \equiv\left(c_{i n}\right)_{i=1}^{n_{c}}, \quad c_{t}=\left(c_{i t}\right)_{i=1}^{n_{c}}, \quad c_{o} \equiv\left(c_{i o}\right)_{i=1}^{n_{c}},
\end{aligned}
$$

we may write the kinematic constraints as the system of linear equations

$$
\left[\begin{array}{l}
a_{n} \\
a_{t} \\
a_{o}
\end{array}\right]=A\left[\begin{array}{l}
c_{n} \\
c_{t} \\
c_{o}
\end{array}\right]+\left[\begin{array}{l}
b_{n} \\
b_{t} \\
b_{o}
\end{array}\right],
$$


where the matrix $A$ and vector $b$ contain given data, defined as follows:

$$
\begin{aligned}
\equiv & {\left[\begin{array}{cc}
W_{n}^{T} & J_{n} \\
W_{t}^{T} & J_{t} \\
W_{o}^{T} & J_{o}
\end{array}\right]\left[\begin{array}{cc}
M_{\mathrm{obj}}^{-1} & 0 \\
0 & M_{\text {man }}^{-1}
\end{array}\right]\left[\begin{array}{ccc}
W_{n} & W_{t} & W_{o} \\
J_{n}^{T} & J_{t}^{T} & J_{o}^{T}
\end{array}\right], } \\
{\left[\begin{array}{c}
b_{n} \\
b_{t} \\
b_{o}
\end{array}\right] \equiv } & {\left[\begin{array}{cc}
\dot{W}_{n}^{T} & \dot{J}_{n} \\
\dot{W}_{t}^{T} & \dot{J}_{t} \\
\dot{W}_{o}^{T} & \dot{J}_{o}
\end{array}\right]\left[\begin{array}{c}
\dot{q} \\
-\dot{\theta}
\end{array}\right] } \\
+ & {\left[\begin{array}{cc}
W_{n}^{T} & J_{n} \\
W_{t}^{T} & J_{t} \\
W_{o}^{T} & J_{o}
\end{array}\right]\left[\begin{array}{cc}
M_{\mathrm{obj}}^{-1} & 0 \\
0 & M_{\operatorname{man}}^{-1}
\end{array}\right]\left[\begin{array}{c}
g_{\mathrm{obj}}+h_{\mathrm{obj}} \\
g_{\mathrm{man}}+h_{\mathrm{man}}-\tau
\end{array}\right] . }
\end{aligned}
$$

We refer the reader to [198] for the mechanical interpretation of the entries of the matrix $A$, the vector $b$, and for a description of the generation of these data from realistic contact problems. Here we simply mention that the matrices $M_{\operatorname{man}}$ and $M_{\text {obj }}$ are symmetric positive definite; thus $A$ is symmetric positive semidefinite.

The Signorini nonpenetrating condition stipulates that the normal accelerations are nonnegative and the normal forces are nontensile and that these accelerations and forces are complementary in the following sense:

$$
0 \leq a_{n} \perp c_{n} \geq 0 .
$$

There are no such requirements on the nonnormal accelerations and forces; instead the latter variables must obey Coulomb's friction law which states that for given, positive friction coefficients $\mu_{i}, i=1, \ldots, n_{c}$,

$$
\begin{aligned}
& \mu_{i} c_{i n}\left(v_{i t}, v_{i o}\right)+\sqrt{v_{i t}^{2}+v_{i o}^{2}}\left(c_{i t}, c_{i o}\right)=0 \quad \text { for } i \in \mathcal{S}, \\
& \left.\begin{array}{r}
\mu_{i} c_{i n}\left(a_{i t}, a_{i o}\right)+\sqrt{a_{i t}^{2}+a_{i o}^{2}}\left(c_{i t}, c_{i o}\right)=0 \\
c_{i t}^{2}+c_{i o}^{2} \leq \mu_{i}^{2} c_{i n}^{2}
\end{array}\right\} \text { for } i \in \mathcal{R} .
\end{aligned}
$$

Equations (3.1), (3.4), (3.5), and (3.6) define the dynamic rigid-body contact problem with Coulomb friction. As shown in [151], this problem can be equivalently formulated as a mixed nonlinear complementarity problem of the type (2.2) by (i) eliminating the sliding force variables $\left(c_{i t}, c_{i o}\right), i \in \mathcal{S}$ using equation (3.5), (ii) removing the constraints defining the sliding acceleration variables $\left(a_{i t}, a_{i o}\right), i \in \mathcal{S}$, and (iii) expressing each of the unrestricted rolling variables $\left(a_{i t}, a_{i o}, c_{i t}, c_{i o}\right), i \in \mathcal{R}$ as the difference of two 
nonnegative variables. The resulting complementarity formulation is defined by the variables $\left(a_{n}, a_{\mathcal{R} t}^{ \pm}, a_{\mathcal{R}_{o}}^{ \pm}, c_{n}, a_{\mathcal{R} t}^{ \pm}, a_{\mathcal{R}_{o}}^{ \pm}\right)$and has the following equation form:

$$
\begin{gathered}
{\left[\begin{array}{c}
a_{n} \\
a_{\mathcal{R} t}^{+} \\
a_{\mathcal{R} o}^{+}
\end{array}\right]=B\left[\begin{array}{c}
c_{n} \\
c_{\mathcal{R} t}^{+} \\
c_{\mathcal{R} t}^{-} \\
c_{\mathcal{R} o}^{+} \\
c_{\mathcal{R} o}^{-} \\
a_{\mathcal{R} t}^{-} \\
a_{\mathcal{R} o}^{-}
\end{array}\right]+\left[\begin{array}{c}
b_{n} \\
b_{\mathcal{R} t} \\
b_{\mathcal{R} o}
\end{array}\right],} \\
\min \left(a_{\mathcal{R} t}^{+}, c_{\mathcal{R} t}^{+}\right)=\min \left(a_{\mathcal{R} o}^{+}, c_{\mathcal{R} o}^{+}\right)=0, \\
\min \left(a_{\mathcal{R} t}^{-}, c_{\mathcal{R} t}^{-}\right)=\min \left(a_{\mathcal{R} o}^{-}, c_{\mathcal{R} o}^{-}\right)=0, \\
\min \left(\mu_{\mathcal{R}}^{2} c_{\mathcal{R} n}^{2}-\left(c_{\mathcal{R} t}^{+}-c_{\mathcal{R} t}^{-}\right)^{2}-\left(c_{\mathcal{R} o}^{+}-c_{\mathcal{R} o}^{-}\right)^{2},\left(a_{\mathcal{R} t}^{+}-a_{\mathcal{R} t}^{-}\right)^{2}+\left(a_{\mathcal{R} o}^{+}-a_{\mathcal{R} o}^{-}\right)^{2}\right)=0,
\end{gathered}
$$

where in the next-to-last equation, the circle $\circ$ denotes the Hadamard product of two vectors, and in the last equation, the subscript $\mathcal{R}$ is an abuse of notation with the meaning of "for each $i \in \mathcal{R}$." The matrix $B$ is obtained from the matrix $A$ in (3.2) after the aforementioned reduction steps; see the reference for details. We point out that unlike $A, B$ is neither symmetric nor positive semidefinite.

A modification of the above model leads to a standard linear complementarity problem. The modified model replaces the (nonlinear) friction cone constraint (3.6) at the rolling contacts with the following friction pyramid constraints:

$$
\left.\begin{array}{r}
\mu_{i} c_{i n}\left(\left|a_{i t}\right|,\left|a_{i o}\right|\right)+\left(a_{i t} c_{i t}, a_{i o} c_{i o}\right)=0 \\
\max \left(\left|c_{i t}\right|,\left|c_{i o}\right|\right) \leq \mu_{i} c_{i n}
\end{array}\right\} \text { for } i \in \mathcal{R} .
$$

The resulting linear complementarity problem is as follows:

$$
\begin{array}{r}
{\left[\begin{array}{c}
a_{\mathcal{S} n} \\
a_{\mathcal{R} n} \\
a_{\mathcal{R} t}^{+} \\
a_{\mathcal{R} o}^{+} \\
s_{\mathcal{R} t}^{-} \\
s_{\mathcal{R} o}^{-}
\end{array}\right]=M\left[\begin{array}{c}
c_{\mathcal{S} n} \\
c_{\mathcal{R} n} \\
s_{\mathcal{R} t}^{+} \\
s_{\mathcal{R} o}^{+} \\
a_{\mathcal{R} t}^{-} \\
a_{\mathcal{R} o}^{-}
\end{array}\right]+\left[\begin{array}{c}
b_{\mathcal{S} n} \\
b_{\mathcal{R} n} \\
b_{\mathcal{R} t} \\
b_{\mathcal{R} o} \\
0 \\
0
\end{array}\right],} \\
\min \left(a_{\mathcal{R} n}, c_{\mathcal{R} n}\right)=0, \\
\min \left(a_{\mathcal{S} n}, c_{\mathcal{S} n}\right)=0, \\
\min \left(a_{\mathcal{R} t}^{+}, s_{\mathcal{R} t}^{+}\right)=\min \left(a_{\mathcal{R} o}^{+}, s_{\mathcal{R} o}^{+}\right)=0, \\
\min \left(a_{\mathcal{R} t}^{-}, s_{\mathcal{R} t}^{-}\right)=\min \left(a_{\mathcal{R} o}^{-}, s_{\mathcal{R} o}\right)=0,
\end{array}
$$


where

$$
M \equiv\left[\begin{array}{cccccc}
\left(M_{n n}\right)_{\mathcal{S S}} & \left(M_{n n}\right)_{\mathcal{S R}} & \left(A_{n t}\right)_{\mathcal{S R}} & \left(A_{n o}\right)_{\mathcal{S R}} & 0 & 0 \\
\left(M_{n n}\right)_{\mathcal{R S}} & \left(M_{n n}\right)_{\mathcal{R} \mathcal{R}} & \left(A_{n t}\right)_{\mathcal{R} \mathcal{R}} & \left(A_{n o}\right)_{\mathcal{R R}} & 0 & 0 \\
\left(M_{t n}\right)_{\mathcal{R S}} & \left(M_{t n}\right)_{\mathcal{R R}} & \left(A_{t t}\right)_{\mathcal{R R}} & \left(A_{t o}\right)_{\mathcal{R R}} & I & 0 \\
\left(M_{o n}\right)_{\mathcal{R S}} & \left(M_{o n}\right)_{\mathcal{R} \mathcal{R}} & \left(A_{o t}\right)_{\mathcal{R R}} & \left(A_{o o}\right)_{\mathcal{R R}} & 0 & I \\
0 & 2 U_{\mathcal{R}} & -I & 0 & 0 & 0 \\
0 & 2 U_{\mathcal{R}} & 0 & -I & 0 & 0
\end{array}\right]
$$

with $U_{\mathcal{R}}$ being the diagonal matrix with diagonal entries $\mu_{i}, i \in \mathcal{R}$. We refer to [151] for the explicit expressions of the other block submatrices in $M$. We point out here that when the matrix $A$ is positive definite and all frictional coefficients $\mu_{i}$ are sufficiently small, $M$ is copositive and belongs to the class of $\mathbf{P}_{0}$-matrices; moreover, the above LCP can be solved by the classical Lemke method and a feasible interior point method. Details and numerical results can be found in [198]. An extension of the models described herein to include soft finger contacts is described in [152]. Related work on elastic body, soft finger contact with friction can be found in [2] and [145, Chapter 14].

Alternative rigid-body frictional contact models that allow for impulsive forces have recently been proposed in $[6,189]$; these models lead to complementarity problems that always have a solution. Further models involving impulses can be found in the recent text [159].

3.1.2. A discretized large displacement frictional contact problem. The following formulation of a discretized, three-dimensional, time incremental, large displacement contact problem with Coulomb friction is based on the discussion in $[94,96,97]$. This problem is the result of a time-space discretization and the numerical integration of a continuum contact problem involving a deformable body and a rigid surface $S$ in $\mathbf{R}^{3}$. It is assumed that this surface $S$ is described by a smooth function $\Psi_{n}: \mathbf{R}^{3} \rightarrow \mathbf{R}$ with the gradient $\nabla \Psi_{n}$ defining the normal fields. We have $x \in S$ if and only if $\Psi_{n}(x)=0$. The motion of the body is restricted to be on or above the surface $S$. The tangential directions of the rigid surface are described by two smooth functions $\Psi_{t}, \Psi_{o}: \mathbf{R}^{3} \rightarrow \mathbf{R}$ satisfying the orthogonal condition:

$$
\nabla \Psi_{n}(x)^{T} \nabla \Psi_{\alpha}(x)=0 \quad \text { for } \alpha \in\{t, o\}
$$

for all $x \in \mathbf{R}^{3}$. Let $M$ denote the number of integrating points in approximating certain surface integrals (these can be thought of as the contact points), and let $N$ denote the total number of displacement degrees of freedom. For each $i=1, \ldots, M$, let $\left(p_{n}^{i}, p_{t}^{i}, p_{o}^{i}\right) \in \mathbf{R}^{3}$ denote the coordinates of the contact force at the $i$ th contact point with respect to the coordinate chart $\left(\Psi_{n}, \Psi_{t}, \Psi_{o}\right)$ on the contact surface $S$. For $\alpha \in\{n, t, o\}$, let $p_{\alpha} \equiv\left(p_{\alpha}^{i}\right)_{i=1}^{M}$ be the vector of contact forces that corresponds to the direction $\alpha$ at the various contact points. Let $u \in \mathbf{R}^{N}$ denote the vector of nodal point displacements, and let $u_{c}$ be the subvector of $u$ that pertains to the contact points; we may write $u=\left(u_{c}, u^{\prime}\right)$ and $u_{c}=\left(u_{c}^{i}\right)_{i=1}$ where each $u_{c}^{i}$ is a three-dimensional vector, with components $\left(u_{c}^{i}\right)_{\alpha}$, for $\alpha \in\{n, t, o\}$. Let $H(u)$ be a vector-valued, displacement dependent function representing the internal forces, and let $F$ be a vector of external forces; both $H(u)$ and $F$ are of dimension $N$. The 
following equation is an equilibration of the contact, external, and internal forces:

$$
H(u)+\left[\begin{array}{c}
\sum_{\alpha \in\{n, t, o\}} \nabla \boldsymbol{\Psi}_{\alpha}\left(u_{c}\right)^{T} p_{\alpha} \\
0
\end{array}\right]=F,
$$

where for each $\alpha$, the boldface function $\boldsymbol{\Psi}_{\alpha}\left(u_{c}\right)$ is the $M$-dimensional vector-valued function with components $\Psi_{\alpha}\left(u_{c}^{i}\right)$ for $i=1, \ldots, M$; thus $\nabla \boldsymbol{\Psi}_{\alpha}\left(u_{c}\right)$ is a block diagonal matrix with blocks consisting of $1 \times 3$ matrices $\left(\nabla \Psi_{\alpha}\left(u_{c}^{i}\right)\right)^{T}$ for $i=1, \ldots, M$. The contact conditions are expressed by the following complementarity restrictions on the normal forces and coordinates: for $i=1, \ldots, M$,

$$
0 \leq p_{n}^{i} \perp \Psi_{n}\left(u_{c}^{i}\right) \leq 0 .
$$

In the references [94, 96, 97], the frictional constraints are described as a variational inequality. Nonpolyhedral friction cones and their polyhedral approximations have been used (similar to the situation described in the last subsection). In what follows, we shall describe both kinds of friction cones. For given $\left(p_{n}, u_{c}\right)$, let

$$
K\left(p_{n}, u_{c}\right) \equiv\left\{\left(\begin{array}{c}
p_{t}^{\prime} \\
p_{o}^{\prime}
\end{array}\right) \in \mathbf{R}^{2 M}: \phi_{i}\left(p_{n}^{i}, p_{t}^{i}, p_{o}^{i}, u_{c}^{i}\right) \leq 0, i=1, \ldots, M\right\}
$$

where

$$
\begin{aligned}
& \phi_{i}\left(p_{n}^{i}, p_{t}^{i}, p_{o}^{i}, u_{c}^{i}\right) \\
& \equiv\left(\frac{\partial \Psi_{t}\left(u_{c}^{i}\right)}{\partial\left(u_{c}^{i}\right)_{t}} p_{t}^{i}+\frac{\partial \Psi_{t}\left(u_{c}^{i}\right)}{\partial\left(u_{c}^{i}\right)_{o}} p_{o}^{i}\right)^{2}+\left(\frac{\partial \Psi_{o}\left(u_{c}^{i}\right)}{\partial\left(u_{c}^{i}\right)} p_{t}^{i}+\frac{\partial \Psi_{o}\left(u_{c}^{i}\right)}{\partial\left(u_{c}^{i}\right)_{o}} p_{o}^{i}\right)^{2}-\mu_{i}^{2}\left(\frac{\partial \Psi_{n}\left(u_{c}^{i}\right)}{\partial\left(u_{c}^{i}\right)_{n}} p_{n}^{i}\right)^{2},
\end{aligned}
$$

where $\mu_{i}>0$ is the friction coefficient at the $i$ th contact point. The Coulomb friction law is the requirement that $\left(p_{t}, p_{o}\right) \in K\left(p_{n}, u_{c}\right)$ and

$$
\left(\begin{array}{c}
\boldsymbol{\Psi}_{t}\left(u_{c}\right)-\boldsymbol{\Psi}_{t}\left(u_{c}^{\text {giv }}\right) \\
\boldsymbol{\Psi}_{o}\left(u_{c}\right)-\boldsymbol{\Psi}_{o}\left(u_{c}^{\text {giv }}\right)
\end{array}\right)^{T}\left(\begin{array}{c}
p_{t}^{\prime}-p_{t} \\
p_{o}^{\prime}-p_{o}
\end{array}\right) \geq 0 \quad \forall\left(p_{t}^{\prime}, p_{o}^{\prime}\right) \in K\left(p_{n}, u_{c}\right),
$$

where $u_{c}^{\text {giv }}$ is a known displacement vector computed at the preceding discretized time. It has been mentioned in [94, section 6] that an alternative inequality which replaces the first parenthesis in (3.11) by

$$
\left(\begin{array}{c}
\nabla \boldsymbol{\Psi}_{t}\left(u_{c}\right)\left(u_{c}-u_{c}^{\mathrm{giv}}\right) \\
\nabla \boldsymbol{\Psi}_{o}\left(u_{c}\right)\left(u_{c}-u_{c}^{\mathrm{giv}}\right)
\end{array}\right)
$$

can also be used to describe the time discretized Coulomb friction law.

Let $\phi\left(p_{n}, p_{t}, p_{o}, u_{c}\right)$ denote the $M$-dimensional function with components

$$
\phi_{i}\left(p_{n}^{i}, p_{t}^{i}, p_{o}^{i}, u_{c}^{i}\right), i=1, \ldots, M .
$$

By introducing multipliers $\lambda_{i}, i=1, \ldots, M$, the inequality (3.11) is equivalent to the following complementarity system:

$$
\left(\begin{array}{c}
\boldsymbol{\Psi}_{t}\left(u_{c}\right)-\boldsymbol{\Psi}_{t}\left(u_{c}^{\text {giv }}\right) \\
\boldsymbol{\Psi}_{o}\left(u_{c}\right)-\boldsymbol{\Psi}_{o}\left(u_{c}^{\text {giv }}\right)
\end{array}\right)+\sum_{i=1}^{M} \lambda_{i}\left(\begin{array}{c}
\nabla_{p_{t}} \boldsymbol{\phi}_{i}\left(p_{n}, p_{t}, p_{o}, u_{c}\right) \\
\nabla_{p_{o}} \boldsymbol{\phi}_{i}\left(P_{n}, p_{t}, p_{o}, u_{c}\right)
\end{array}\right)=0
$$


and

$$
0 \leq \lambda_{i} \perp \phi_{i}\left(p_{n}^{i}, p_{t}^{i}, p_{o}^{i}, u_{c}^{i}\right) \leq 0, \quad i=1, \ldots, M .
$$

The three-dimensional, time incremental, large displacement, frictional contact problem is to find force vectors $\left(p_{n}, p_{t}, p_{o}\right)$ and displacement vector $u=\left(u_{c}, u^{\prime}\right)$ such that $\left(p_{t}, p_{o}\right) \in K\left(p_{n}, u\right)$ and (3.9), (3.10), (3.12), and (3.13) hold. In general this is a highly nonlinear problem; there are three sources of nonlinearity: the displacement function $H$, the coordinate chart $\left(\boldsymbol{\Psi}_{n}, \boldsymbol{\Psi}_{t}, \boldsymbol{\Psi}_{o}\right)$, and the Coulomb friction law. The theory of "quasi-variational inequality problem" [20] can be applied to study the question of solution existence for this problem. However, there remain a lot of theoretical and algorithmic issues that await treatment.

Simplifications of the above model result when one considers the small displacement contact problem coupled with a piecewise linearization of the friction cone. In what follows, we describe a model of this type that leads to a mixed linear complementarity problem. This linearized model is discussed in the cited references also; in particular, the papers $[90,96]$ described some pivotal methods for solving the resulting (linear) complementarity problem.

In the small displacement contact problem, it is assumed that the function $H$ is linear and given by

$$
H(u)=K u,
$$

where $K$ is a symmetric, positive semidefinite stiffness matrix. For simplicity, we assume that $u=u_{c}$. The coordinate functions $\left(\boldsymbol{\Psi}_{n}, \boldsymbol{\Psi}_{t}, \boldsymbol{\Psi}_{o}\right)$ are approximated by their linearization at $u_{c}^{\text {giv }}$; i.e.,

$$
\boldsymbol{\Psi}_{\alpha}\left(u_{c}\right) \approx \boldsymbol{\Psi}_{\alpha}\left(u_{c}^{\mathrm{giv}}\right)+\nabla \boldsymbol{\Psi}_{\alpha}\left(u_{c}^{\mathrm{giv}}\right)\left(u_{c}-u_{c}^{\mathrm{giv}}\right) \equiv g_{\alpha}+C_{\alpha} u_{c} \quad \text { for } \alpha \in\{n, t, o\} .
$$

The friction cone $K\left(p_{n}, u_{c}\right)$ is approximated by a polytope of the type

$$
K^{\operatorname{lin}}\left(p_{n}\right) \equiv \prod_{i=1}^{M}\left\{\left(p_{t}^{i}, p_{o}^{i}\right) \in \mathbf{R}^{2}:\left(\cos a_{i j}\right) p_{t}^{i}+\left(\sin a_{i j}\right) p_{o}^{i}-\mu_{i} p_{n}^{i} \leq 0, j=1, \ldots, r_{i}\right\},
$$

where for each $i=1, \ldots, M, r_{i}$ is positive integer, and

$$
a_{i j} \equiv(j-1) \frac{2 \pi}{r_{i}}, \quad j=1, \ldots, r_{i},
$$

with $r_{i}>0$ being a positive integer. Notice that in $K\left(p_{n}, u_{c}\right)$, there is only one constraint $\phi_{i}\left(p_{n}^{i}, p_{t}^{i}, p_{o}^{i}, u_{c}^{i}\right) \leq 0$ for each index $i$; this single constraint is replaced by $r_{i}$ constraints:

$$
\left(\cos a_{i j}\right) p_{t}^{i}+\left(\sin a_{i j}\right) p_{o}^{i}-\mu_{i} p_{n}^{i} \leq 0, \quad j=1, \ldots, r_{i} .
$$

In the cited references, this problem has been formulated as a standard LCP. This formulation relies on the assumption that $K$ is positive definite. In what follows, we give a complete formulation of the small-displacement contact problem as a mixed LCP for a general matrix $K$. For each $i=1, \ldots, M$, let $\lambda^{i} \in \mathbf{R}^{r_{i}}$ denote the multiplier vector associated with the constraints (3.14); also define two $r_{i}$-dimensional vectors:

$$
G_{t}^{i} \equiv\left(\begin{array}{c}
\cos a_{i 1} \\
\vdots \\
\cos a_{i r_{i}}
\end{array}\right) \text { and } \quad G_{0}^{i} \equiv\left(\begin{array}{c}
\sin a_{i 1} \\
\vdots \\
\sin a_{i r_{i}}
\end{array}\right) \text {. }
$$


Finally let $e^{i}$ denote the $r_{i}$-dimensional vector of all ones. The three-dimensional, small displacement, frictional contact problem is to find force vectors $\left(p_{n}, p_{t}, p_{o}\right)$, a displacement vector $u$, and multiplier vectors $\lambda^{i}, i=1, \ldots, M$ such that

$$
\left.\begin{array}{rl}
K u+ & C_{n}^{T} p_{n}+C_{t}^{T} p_{t}+C_{o}^{T} p_{o}=F \\
0 & \leq p_{n} \perp g_{n}+C_{n} u \leq 0, \\
0 \leq \lambda^{i} & \perp G_{t}^{i} p_{t}^{i}+G_{o}^{i} p_{o}^{i}-\mu_{i} p_{n}^{i} e^{i} \leq 0 \\
\left(C_{t} u\right)_{i}+\left(G_{t}^{i}\right)^{T} \lambda^{i}=0 \\
\left(C_{o} u\right)_{i}+\left(G_{o}^{i}\right)^{T} \lambda^{i}=0
\end{array}\right\} i=1, \ldots, M .
$$

Sufficient conditions are known under which the above problem has a solution [98]; however, conditions for solution existence that are both necessary and sufficient are not available now. Moreover, it would be useful to have algorithms that are guaranteed to compute a solution to the problem whenever it exists. These issues and several others remain to be investigated.

3.2. Structural mechanics problems. The application of mathematical programming (particularly, complementarity) theory and methods to analyze elastoplastic structures dates back to the late 1960s and early 1970s. Maier and his colleagues from the Italian school $[37,119,120,121]$ were the pioneers in this approach. Kaneko $[85,86,87]$ and Wakefield and Tin-Loi $[201,202]$ focused on the linear complementarity approach. The recent papers $[28,122,192,193,194]$ employed the nonlinear complementarity problem to model nonlinear hardening laws and static-kinematic relations in these structural problems.

In what follows, we consider a discretized, elastoplastic structure undergoing deformation when it is acted upon by external loads. Our discussion follows the reference [194], which concerns a semirigidly connected plane structure in the presence of both material and geometrical nonlinearities. This structure is discretized into $n$ finite elements. For each element $m \in\{1, \ldots, n\}$, let $Q^{m}, q^{m} \in \mathbf{R}^{3}$ denote, respectively, the (unknown) vectors of generalized stresses and strains and $u^{m} \in \mathbf{R}^{6}$ the (unknown) vector of nodal displacements. Let $F^{m} \in \mathbf{R}^{6}$ be a vector of external forces acting on the $m$ th member element which is of length $L_{m}$ and at angle $\theta_{m}$ to some horizontal reference axis; $F^{m}, L_{m}$, and $\theta_{m}$ are given. Let

$$
c_{m} \equiv \cos \theta_{m} \quad \text { and } \quad s_{m} \equiv \sin \theta_{m} .
$$

It is convenient to define two matrices

$$
A^{m} \equiv\left[\begin{array}{ccc}
c_{m} & -s_{m} / L_{m} & -s_{m} / L_{m} \\
s_{m} & c_{m} / L_{m} & c_{m} / L_{m} \\
0 & 1 & 0 \\
-c_{m} & s_{m} / L_{m} & s_{m} / L_{m} \\
-s_{m} & -c_{m} / L_{m} & -c_{m} / L_{m} \\
0 & 0 & 1
\end{array}\right] \quad \text { and } \quad A_{\pi}^{m} \equiv\left[\begin{array}{cc}
c_{m} & -s_{m} \\
s_{m} & c_{m} \\
0 & 0 \\
-c_{m} & s_{m} \\
-s_{m} & -c_{m} \\
0 & 0
\end{array}\right]
$$

We introduce the auxiliary displacement vector

$$
\delta^{m}=\left[\begin{array}{c}
\delta_{n}^{m} \\
\delta_{t}^{m}
\end{array}\right] \equiv\left(A_{\pi}^{m}\right)^{T} u^{m} .
$$


In terms of $\delta^{m}$, we define the angle of member chord rotation and the length of deformed member chord:

$$
\rho_{m} \equiv \arctan \frac{\delta_{t}^{m}}{L_{m}-\delta_{n}^{m}} \quad \text { and } \quad L_{m}^{\prime} \equiv \sqrt{\left(L_{m}-\delta_{n}^{m}\right)^{2}+\left(\delta_{t}^{m}\right)^{2}} .
$$

Let

$$
\begin{gathered}
c_{m}^{\prime} \equiv \cos \rho_{m} \quad \text { and } \quad s_{m}^{\prime} \equiv \sin \rho_{m} \\
Z^{m} \equiv\left[\begin{array}{ccc}
1-c_{m}^{\prime} & -s_{m}^{\prime} / L_{m}^{\prime} & -s_{m}^{\prime} / L_{m}^{\prime} \\
s_{m}^{\prime} & 1 / L_{m}-c_{m}^{\prime} / L_{m}^{\prime} & 1 / L_{m}-c_{m}^{\prime} / L_{m}^{\prime}
\end{array}\right] .
\end{gathered}
$$

Finally, we define

$$
\left(C^{m}\right)^{T} \equiv A^{m}-A_{\pi}^{m} Z^{m} .
$$

Notice that through the relation (3.16), the matrix $C^{m}$ is a function of the displacement vector $u^{m}$.

Simple equilibrium considerations in the deformed state lead to the following balancing equation of forces and stresses:

$$
F^{m}=\left(C^{m}\right)^{T} Q^{m} .
$$

The element kinematic relations between member deformations $q^{m}$ and nodal displacements $u^{m}$ can be written in the form

$$
\left(A^{m}\right)^{T} u^{m}=q^{m}+\left[\begin{array}{c}
\delta_{n}^{m}-q_{1}^{m} \\
\delta_{t}^{m} / L_{m}-\rho_{m} \\
\delta_{t}^{m} / L_{m}-\rho_{m}
\end{array}\right] .
$$

The expressions (3.17) and (3.18) are the static-kinematic equations of the structural model at each member element. We next describe the constitutive laws; these are summarized by the following equations:

$$
\begin{gathered}
q^{m}=e^{m}+p^{m}, \\
Q^{m}=S^{m} e^{m}+R_{b}^{m}, \\
p^{m}=N^{m} \lambda^{m}, \\
\phi^{m}=\left(N^{m}\right)^{T} Q^{m}-H^{m} \lambda^{m}-R^{m}, \\
\min \left(-\phi^{m}, \lambda^{m}\right)=0 .
\end{gathered}
$$

We refer to [194] for a detailed explanation of these equations. Here a brief description is given. Equation (3.19) expresses the total element strains $q^{m}$ as the sum of elastic $e^{m}$ and plastic $p^{m}$ strains; $S^{m}$ is a symmetric, but not necessarily positive semidefinite, matrix of element elastic stiffnesses which are nonlinear functions of the element axial force $Q_{1}^{m}$ and involve some constants pertaining to the semirigid connections at the two ends of the element; $R_{b}^{m}$ accounts for the "bowing" effect and is a three-dimensional 
vector with the last two components equal to zero and the first component being a highly nonlinear function of $Q_{1}^{m}$. Thus (3.20) is in fact a nonlinear equation. Relations (3.21)-(3.23) describe the plastic holonomic (path-independent) constitutive laws as introduced by Maier [119]. It is assumed in these laws that the plastic strains $p^{m}$ are linear functions of the plastic multipliers $\lambda^{m}$, where $N^{m}$ is a constant matrix containing the unit outward normals to the piecewise linearized yield hyperplanes; $\phi^{m}$ is the yield function that is restricted to be nonpositive and complementarity to the multiplier vector $\lambda^{m}$; in essence complementarity in this context stipulates that yielding can only occur for a stress point on the yield surface and implies that no local loading is allowed (i.e., $\phi_{i}^{m}<0$ and $\lambda_{i}^{m}>0$ is not acceptable). Finally, the matrix $H^{m}$ (which is constant and positive semidefinite) and the constant vector $R^{m}$ together define a linear hardening law.

From equations (3.18)-(3.21), we can eliminate and substitute the variables $q^{m}$, $e^{m}, Q^{m}$, and $p^{m}$ into equations (3.17), (3.22), and (3.23); after some algebraic manipulations, we obtain the following mixed nonlinear complementarity system for the member element $m$ :

$$
\begin{gathered}
{\left[\begin{array}{c}
-\phi^{m} \\
0
\end{array}\right]=\left[\begin{array}{cc}
K_{\lambda \lambda}^{m} & K_{\lambda u}^{m} \\
K_{u \lambda}^{m} & K_{u u}^{m}
\end{array}\right]\left[\begin{array}{c}
\lambda^{m} \\
u^{m}
\end{array}\right]+\left[\begin{array}{c}
\left(N^{m}\right)^{T} \\
-\left(C^{m}\right)^{T}
\end{array}\right]\left(S^{m} R_{q}^{m}-R_{b}^{m}\right)+\left[\begin{array}{c}
R^{m} \\
-F^{m}
\end{array}\right],} \\
\min \left(-\phi^{m}, \lambda^{m}\right)=0
\end{gathered}
$$

where

$$
\left[\begin{array}{ll}
K_{\lambda \lambda}^{m} & K_{\lambda u}^{m} \\
K_{u \lambda}^{m} & K_{u u}^{m}
\end{array}\right] \equiv\left[\begin{array}{cc}
\left(N^{m}\right)^{T} S^{m} N^{m}+H^{m} & -\left(N^{m}\right)^{T} S^{m} C^{m} \\
-\left(C^{m}\right)^{T} S^{m} N^{m} & \left(C^{m}\right)^{T} S^{m} C^{m}
\end{array}\right],
$$

and

$$
R_{q}^{m} \equiv C^{m} u^{m}-q^{m}
$$

Although written in the form similar to that of a mixed linear complementarity problem, the above system is actually a highly nonlinear problem because of the nonlinear dependence of the matrix functions $S^{m}$ and $C^{m}$ on the variables $u^{m}$ and $Q_{1}^{m}$. One advantage of this form is that it facilitates the application of a particular iterative algorithm as described in [194].

The overall structural model is derived from the above element models and can be stated as the following mixed NCP:

$$
\begin{gathered}
{\left[\begin{array}{c}
-\Phi \\
0
\end{array}\right]=\left[\begin{array}{cc}
K_{\lambda \lambda} & K_{\lambda u} \\
K_{u \lambda} & K_{u u}
\end{array}\right]\left[\begin{array}{l}
\Lambda \\
\boldsymbol{u}
\end{array}\right]+\left[\begin{array}{c}
N^{T} \\
-C^{T}
\end{array}\right]\left(S R_{q}-R_{b}\right)+\left[\begin{array}{c}
R \\
-F
\end{array}\right],} \\
\min (-\Phi, \Lambda)=0
\end{gathered}
$$

where

$$
K_{\lambda \lambda} \equiv N^{T} S N+H, \quad K_{u u} \equiv C^{T} S C, \quad K_{u \lambda}=K_{\lambda u}^{T} \equiv-C^{T} S N,
$$

the matrices $S, N$, and $H$ are block diagonal matrices with diagonal blocks $S^{m}, N^{m}$, and $H^{m}$, respectively, and the vectors $\Phi, \Lambda, R, R_{q}$, and $R_{b}$ are concatenations of the element vectors $\phi^{m}, \lambda^{m}, R^{m}, R_{q}^{m}$, and $R_{b}^{m}$, respectively. The vector $\boldsymbol{u}$ consists of 


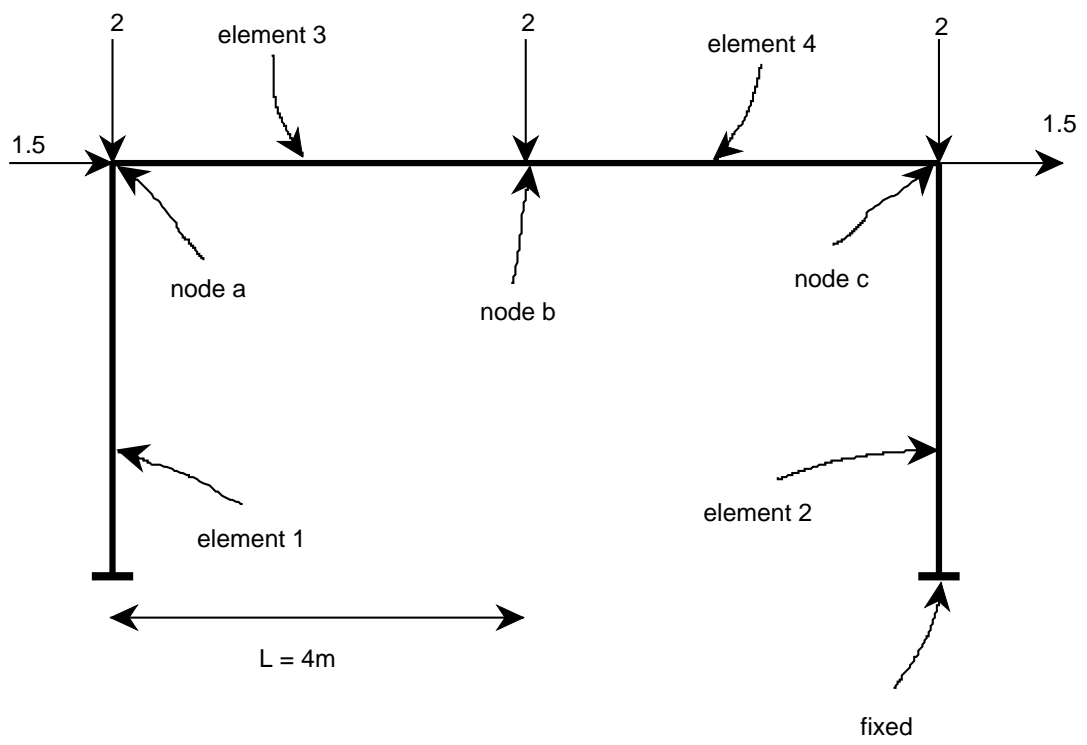

FIG. 3.1. Four-element structure with loading.

the nodal displacements of the structure; the $m$ th element displacement vector $u^{m}$ can be extracted from $\boldsymbol{u}$ according to the particular structure and the finite element discretization. The matrix $C$ is assembled from the element matrices $C^{m}$ resulting in the vector $C \boldsymbol{u}$ being the concatenation of the element vectors $C^{m} u^{m}$.

To more fully explain the assembly outlined above, we need to focus on a particular structure. In Figure 3.1, a simple four element structure is shown that was communicated to us by Tin-Loi. This three-dimensional structure has four elements, each of length 4 meters. The bottom ends of elements 1 and 2 are fixed to the ground. The nodes a, b, and c represent places where external forces are applied as shown in the figure. The nodal displacements are $\left(u_{1}, u_{2}, u_{3}\right),\left(u_{4}, u_{5}, u_{6}\right)$, and $\left(u_{7}, u_{8}, u_{9}\right)$ for nodes a, b, and c, respectively, and together these comprise the vector $\boldsymbol{u}=\left(u_{1}, \ldots, u_{9}\right)$. The element displacements, $u^{1}, u^{2}, u^{3}$, and $u^{4}$ are thus given by

$$
u^{1}=\left[\begin{array}{c}
\cdot \\
\cdot \\
\cdot \\
u_{1} \\
u_{2} \\
u_{3}
\end{array}\right], u^{2}=\left[\begin{array}{c}
\cdot \\
\cdot \\
\cdot \\
u_{7} \\
u_{8} \\
u_{9}
\end{array}\right], u^{3}=\left[\begin{array}{c}
u_{1} \\
u_{2} \\
u_{3} \\
u_{4} \\
u_{5} \\
u_{6}
\end{array}\right], u^{4}=\left[\begin{array}{c}
u_{4} \\
u_{5} \\
u_{6} \\
u_{7} \\
u_{8} \\
u_{9}
\end{array}\right] .
$$

To construct the matrix $C$ from the element matrices $C^{m}$, the following relationship is used:

$$
C \boldsymbol{u}=\left[\begin{array}{l}
C^{1} u^{1} \\
C^{2} u^{2} \\
C^{3} u^{3} \\
C^{4} u^{4}
\end{array}\right]
$$


Thus in the above example

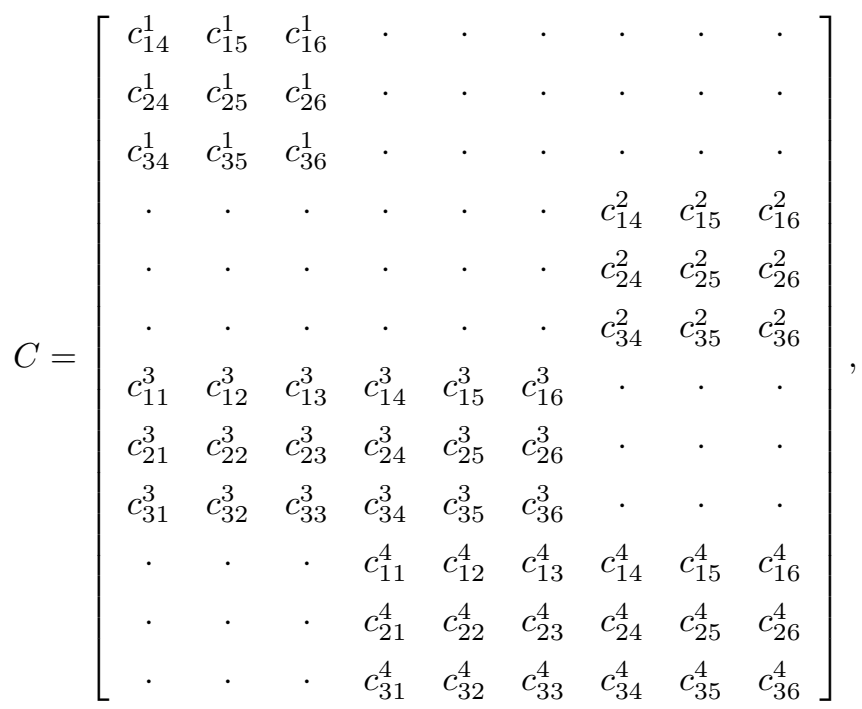

where $c_{i j}^{m}$ refers to the $(i, j)$ element of $C^{m}$.

3.3. Structural design problems as MPECs. Many optimal design problems of discrete or discretized structures in unilateral contact lead to MPECs. The recent doctoral thesis [157] treats several important frictionless contact, truss topology optimization problems which, due to the special first-level objective functions, have an equivalent saddle-point formulation. Extensive review and references are also contained in this thesis. Design problems with friction and more general first-level objective functions lead to two-level optimization problems with complementarity constraints that cannot be formulated as saddle-point problems; some articles that discuss these applications and their numerical solution include $[27,107,106,146$, $170,185,186]$. In what follows, we give the formulation of a minimum weight design problem in truss topology optimization with unilateral, frictionless contact.

The state equations of this design problem are obtained from the system (3.15) by letting $\mu=0$ (frictionless contact); the stiffness matrix $K$ is a function of the design variable $t \in \mathbf{R}^{m}$ which is the vector of bar volumes. A common assumption is

$$
K(t) \equiv \sum_{i=1}^{m} t_{i} K_{i}
$$

where each $K_{i}$ is a symmetric positive definite matrix. The minimum weight design problem can now be defined as follows:

$$
\begin{array}{ll}
\text { minimize } & w\left(u, p_{n}, t\right) \\
\text { subject to } & t \in \mathcal{T}, \\
& h\left(u, p_{n}, t\right) \leq 0, \\
& K(t) u+C_{n}^{T} p_{n}=F, \\
& 0 \leq p_{n} \perp g_{n}+C_{n} u \leq 0,
\end{array}
$$

where $w$ is a generalized weight function, $\mathcal{T}$ is the constraint set of the variable $t$ of bar volumes (examples of $\mathcal{T}$ include $\left\{t \in \Re_{+}^{m}: \sum_{i=1}^{m} t_{i}=V\right\}$, where $V>0$ is the 
given total volume of the bars and $\left\{t \in \Re^{m}: \bar{t} \geq t \geq \underline{t}\right\}$, where $\bar{t}>\underline{t}>0$ are upper and lower bounds on the volume of the bars, respectively), and usual instances of the constraint function $h$ are bounds on displacements and stresses (in which case $h$ will be independent of $p_{n}$ ) and/or bounds on contact forces. Clearly, this problem is an instance of the MPEC with mixed linear complementarity constraints; $t$ is the upper-level variable and $\left(u, p_{n}\right)$ are the lower-level variables.

3.4. Nonlinear obstacle problems. Many obstacle problems in mathematical physics naturally lead to complementarity problems. The monograph [171] gives a detailed account of the applicability of variational inequalities to these obstacle problems. In this context, the complementarity condition is intimately related to the notion of a free boundary; indeed, part of the objective of solving an obstacle problem is to determine this so-called free boundary.

Quadratic programming and linear complementarity methods for solving certain obstacle problems have been well documented [32, 133]. Obstacle problems that lead to (mixed) NCPs include the obstacle Bratu problem [77, 130], the obstacle von Kármán problem [209] and [144, Chapter 7], and several related problems in nonlinear elastic beam theory [62]. Typically, these problems are formulated in infinite-dimensional function spaces and their discretizations could easily lead to finite-dimensional problems of very large size. Numerical solution of the discretized obstacle Bratu problem is discussed in the two cited references and in several recent papers $[24,41,59]$. In what follows, we give the formulation of the obstacle von Kármán problem as given in [144, 209].

The von Kármán equations are of fundamental importance in the theory of thin elastic plates. The points of the plate are given a fixed, right-handed Cartesian coordinate system $0 x_{1} x_{2} x_{3}$. The middle plane of the undeformed plate, which is assumed to have a constant thickness $h$, coincides with the $0 x_{1} x_{2}$ plane. The points $\left(x_{1}, x_{2}, 0\right)$ of the undeformed plate constitute an open, bounded, connected subset $\Omega \subset \mathbf{R}^{2}$ with a Lipschitz boundary $\partial \Omega$. We denote by $u=\left(u_{1}, u_{2}\right)$ the horizontal and by $\xi$ the vertical displacements of the point $x \in \Omega$. The plate is subjected to a distributed load $(0,0, f), f=f(x) \in \mathcal{L}^{2}(\Omega)$, per unit area of the middle surface. The shape of the obstacle is given by a prescribed strictly concave function $\psi(x) \in \mathcal{H}^{2}(\Omega)$. An example is $\psi\left(x_{1}, x_{2}\right) \equiv-\left(x_{1}^{2}+x_{2}^{2}\right)$. Let

$$
K \equiv \frac{E h^{3}}{12\left(1-\nu^{2}\right)}
$$

be the plate's bending rigidity with $E$ the modulus of elasticity and $\nu \in(0,1 / 2)$ the Poisson ratio.

In the following formulation of the obstacle von Kármán problem, the Einstein summation convention with respect to a repeated index is employed: i.e., we write $a_{i} b_{i j}$ to mean $\sum_{i} a_{i} b_{i j}$. Other notation used is as follows: for a scalar-valued function $\phi\left(x_{1}, x_{2}\right), \phi_{, i} \equiv \partial \phi / \partial x_{i} ; \partial \phi / \partial \boldsymbol{n}=(\nabla \phi)^{T} \boldsymbol{n}$ is the directional derivative of $\phi$ in the direction of the outward unit normal vector $\boldsymbol{n}$ to $\partial \Omega$; for a vector-valued function $F\left(x_{1}, x_{2}\right), F_{i, j} \equiv \partial F_{i} / \partial x_{j}$; finally $\Delta \Delta$ is the biharmonic operator with $\Delta \equiv \sum_{i} \partial^{2} / \partial x_{i}^{2}$ being the Laplace operator.

The obstacle von Kármán problem is to find two functions $u\left(x_{1}, x_{2}\right)$ and $\xi\left(x_{1}, x_{2}\right)$ satisfying the following system of partial differential equations and inequalities as well as the given boundary conditions:

$$
K \Delta \Delta \xi-h\left(\sigma_{\alpha \beta} \xi_{, \beta}\right)_{, \alpha} \geq f(x) \quad \text { in } \Omega,
$$




$$
\begin{array}{cc}
\left(\sigma_{\alpha \beta}\right)_{, \beta}=0 & \text { in } \Omega, \\
\xi(x) \geq \psi(x) & \text { in } \Omega, \\
\left(K \Delta \Delta \xi-h\left(\sigma_{\alpha \beta} \xi_{, \beta}\right)_{, \alpha}-f(x)\right)(\xi(x)-\psi(x))= & 0 \text { in } \Omega, \\
\xi(x), u_{\alpha}, \frac{\partial \xi}{\partial \boldsymbol{n}} \quad \text { all given } & \text { on } \partial \Omega,
\end{array}
$$

where $\sigma \equiv\left(\sigma_{\alpha \beta}\right)$ and $\varepsilon \equiv\left(\varepsilon_{\alpha \beta}\right)$ denote, respectively, the stress and strain tensor in the plane of the plate that are linked by the elasticity tensor $C \equiv\left(C_{\alpha \beta \gamma \delta}\right)$; i.e.,

$$
\sigma_{\alpha \beta}=C_{\alpha \beta \gamma \delta}\left(\varepsilon_{\gamma \delta}(u)+\frac{1}{2} \xi_{, \gamma} \xi_{, \delta}\right)
$$

with

$$
\varepsilon_{\gamma \delta}(u) \equiv \frac{1}{2}\left(u_{\gamma, \delta}+u_{\delta, \gamma}\right)=\frac{1}{2}\left(\frac{\partial u_{\gamma}}{\partial x_{\delta}}+\frac{\partial u_{\delta}}{\partial x_{\gamma}}\right) .
$$

In general, each component $C_{\alpha \beta \gamma \delta} \in \mathcal{L}^{\infty}(\Omega)$ and the tensor $C$ satisfies the symmetry and ellipticity properties; i.e.,

$$
C_{\alpha \beta \gamma \delta}=C_{\beta \alpha \gamma \delta}=C_{\gamma \delta \alpha \beta},
$$

and there exists a constant $c>0$ such that for all symmetric matrices $\varepsilon=\left(\varepsilon_{\alpha \beta}\right)$,

$$
C_{\alpha \beta \gamma \delta} \varepsilon_{\alpha \beta} \varepsilon_{\gamma \delta} \geq c \varepsilon_{\alpha \beta} \varepsilon_{\alpha \beta}
$$

For an isotropic plate, $C$ is a constant tensor and has the form

$$
C_{\alpha \beta \lambda \mu}=\frac{E}{2\left(1-\nu^{2}\right)}\left((1-\nu)\left(\delta_{\alpha \lambda} \delta_{\beta \mu}+\delta_{\alpha \mu} \delta_{\beta \lambda}\right)+2 \nu \delta_{\alpha \beta} \delta_{\lambda \mu}\right),
$$

where $\delta_{\alpha \beta}$ is the standard Kroneker delta notation.

Upon discretization, problem (3.24)-(3.28) leads to a large, mixed NCP where the defining function is a multivariate cubic polynomial. To date, there is no reported numerical results for solving this highly complex complementarity problem.

A simplified one-dimensional version of the above problem was communicated to the authors by David Gao. This is an obstacle large deformed beam problem with boundary conditions; it pertains to the computation of a scalar function $w(x)$ satisfying

$$
\begin{array}{cl}
w^{\prime \prime \prime \prime}-c\left(w^{\prime}\right)^{2} w^{\prime \prime} \geq f & \forall x \in(0, L), \\
w(x) \geq \psi(x) & \forall x \in(0, L), \\
\left(w^{\prime \prime \prime \prime}-c\left(w^{\prime}\right)^{2} w^{\prime \prime}-f\right)(w-\psi)=0 & \forall x \in(0, L),
\end{array}
$$

and given boundary conditions at $x=0, L$, where $c$ and $L$ are two given positive scalars, $f(x)$ and $\psi(x)$ are given functions, and ' denotes standard one-dimensional derivative.

3.5. Elastohydrodynamic lubrication problems. The theory of elastohydrodynamic lubrication (EHL) addresses the important effects of elastic deformation and pressure-sensitive viscosity on the operational characteristics of lubricated surfaces [140]. Previous work in this area by complementarity methods has been documented in $[33,35,41,78,104,105,137,138,139,140,141,190]$. As noted by a 
referee, it was reported in [29] that the nonlinear programming package LANCELOT was unable to solve the complementarity problems arising from these applications.

Typically, the defining equations of an EHL problem are of the following kinds: a Reynolds equation that relates the lubricant pressure and the fluid film thickness, when such a film is present; a linear integral equation that explicitly defines the film thickness as a displacement of points on the bearing surfaces due to the lubricant pressure; some equilibrium conditions balancing the lubricant pressure and applied loads; an equation relating the gap between the contact surfaces and contact pressure, when solid-to-solid contact is allowed to coexist. The reference [139] discusses a mixed lubrication problem that combines solid-to-solid contact and hydrodynamic lubrication. In such a problem, complementarity arises in two ways. One source for complementarity is due to the cavitation phenomenon. That is, a fluid film may not develop in some portion of the load-bearing surface. In the cavitated region, the pressure is zero and Reynolds equation fails; in the lubricated region, the pressure is positive and Reynolds equation applies. The identification of the unknown (also called free) boundary separating these two regions is an important issue in an EHL study. Another source where complementarity arises is due to solid-to-solid contact at points where no fluid film is formed; at such contact points, contact (instead of lubricant) pressure is developed and this is governed by the laws of solid elasticity. The resulting complementarity problem is of the vertical type.

The complete formulation of a two-dimensional EHL problem as a finite-dimensional (mixed) complementarity problem is very complicated. This is because partial differential and multiple integration operators are involved. Although the numerical solution of such a problem by a complementarity algorithm of the pivotal type has been reported, much work remains to be done in order for the recent iterative methods to be applicable. A one-dimensional problem is formulated as a GAMS model in [40].

3.6. Traffic equilibrium problems. Underlying all models used for analysis of traffic congestion, there is a transportation network given by a set of nodes $\mathcal{N}$ and a set of $\operatorname{arcs} \mathcal{A}$. The models are used to predict the steady-state volume of traffic on this network. It is usually assumed that drivers compete noncooperatively for the resources of the network in an attempt to minimize their costs, where the cost of traveling along a given arc $a \in \mathcal{A}$ is a nonlinear function $c_{a}(f)$ of the total flow vector $f$ with components $f_{b}, b \in \mathcal{A}$. Let $c(f)$ denote the vector with components $c_{a}(f)$, $a \in \mathcal{A}$. There are two subsets of $\mathcal{N}$ that represent the set of origin nodes $\mathcal{O}$ and destination nodes $\mathcal{D}$, respectively. The set of origin-destination (O-D) pairs is a given subset $\mathcal{W}$ of $\mathcal{O} \times \mathcal{D}$; associated with each such pair is a travel demand that represents the required flow from the origin node to the destination node.

There are two techniques used for generating models of traffic congestion on the given network. The first model is based on considering all the paths between the O-D pairs, and the second uses a multicommodity formulation representing each origin or destination node as a different commodity. Both of these formulations use the Wardropian characterizations of equilibria [205] that may be considered as a special case of a Nash equilibrium (see subsection 4.3 and [75]). Many papers have discussed the formulation and solution of traffic equilibrium problems by the methods of complementarity and variational inequality as well as the applications of these problems in urban planning; see [49, 50, 54, 118, 181].

3.6.1. A path-based formulation. The description of this formulation follows [53]. For each $w \in \mathcal{W}$, let $\mathcal{P}_{w}$ represent the set of paths connecting the O-D pair $w$, and let $\mathcal{P}$ represent the set of all paths joining all O-D pairs of the network. Let $\xi_{p}$ 
denote the flow on path $p \in \mathcal{P}$; let $\gamma_{p}(\xi)$ be the cost of flow on this path which is a function of the path flow vector $\xi$. Let $\Delta$ be the arc-path incidence matrix with entries

$$
\delta_{a p} \equiv \begin{cases}1 & \text { if path } p \in \mathcal{P} \text { traverses arc } a \in \mathcal{A} \\ 0 & \text { otherwise. }\end{cases}
$$

It is clear that $f$ and $\xi$ are related by

$$
f=\Delta \xi .
$$

When the path cost $\gamma_{p}(\xi)$ on each path, $p$ is assumed to be the sum of the arc costs on all the arcs traversed by $p$; that is, if

$$
\gamma(\xi) \equiv \Delta^{T} c(f),
$$

the model is called additive. Finally, we introduce variables $\tau_{w}$ that depict the minimum transportation cost (or time) between O-D pair $w \in \mathcal{W}$. The travel demand between O-D pair $w$ is assumed to be a function $d_{w}(\tau)$ of the vector $\tau$ in the path formulation. The model is called a fixed-demand model if each $d_{w}(\tau)$ is a constant function; the general model is often called the elastic demand model.

The Wardrop equilibrium principle [205] states that each driver will choose the minimum cost path between every origin destination pair, and through this process the paths that are used will have equal cost; paths with costs higher than the minimum will have no flow. Mathematically, this principle can be phrased succinctly as

$$
0 \leq \gamma_{p}(\xi)-\tau_{w} \perp \xi_{p} \geq 0 \quad \forall w \in \mathcal{W}, p \in \mathcal{P}_{w} .
$$

The demand is satisfied if

$$
\sum_{p \in \mathcal{P}_{w}} \xi_{p} \geq d_{w}(\tau) \quad \forall w \in \mathcal{W},
$$

and the equilibrium condition of zero excess demand can be stated as follows,

$$
0 \leq \sum_{p \in \mathcal{P}_{w}} \xi_{p}-d_{w}(\tau) \perp \tau_{w} \geq 0 \quad \forall w \in \mathcal{W} .
$$

Conditions (3.32) and (3.33) clearly define a nonlinear complementarity problem with $(\xi, \tau)$ as the variables.

For networks of reasonable size, the enumeration of all paths connecting elements of $\mathcal{W}$ is prohibitive, if there are many O-D pairs. Thus the above path-flow formulation is not suitable for direct computational use. Nevertheless, there are path-generation schemes $[56,76,154]$ that utilize this formulation and generate the paths only if they are needed. The alternative multicommodity formulation to be discussed below completely removes the necessity of enumerating the paths.

3.6.2. A multicommodity formulation. In this alternative formulation of the traffic equilibrium problem, a commodity is associated with each destination node. For simplicity, we assume that each node in $\mathcal{D}$ is a destination. Let $K$ be the cardinality of $\mathcal{D}$. The variable $x=\left(x^{1}, x^{2}, \ldots, x^{K}\right)$ represents the flows of the commodities $1,2, \ldots, K$ with $x_{a}^{k}$ denoting the flow of commodity $k$ on arc $a \in \mathcal{A}$. The variable $t=\left(t^{1}, t^{2}, \ldots, t^{K}\right)$ is composed of components $t_{i}^{k}$ that represent the minimum cost (or time) to deliver commodity $k$ (i.e., to reach destination $k$ ) from node $i$. Associated 
with each pair $(k, i)$ with $k \in \mathcal{D}$ and $i \in \mathcal{N}$ is the travel demand $d_{i}^{k}$, which is a function of the minimum cost vector $t$. For each node $i$, let $\mathcal{A}_{i}^{+}$and $\mathcal{A}_{i}^{-}$denote, respectively, the sets of outgoing and incoming arcs at node $i$. There are two sets of equilibrium conditions. The first represents conservation of flow of commodity $k$ at node $i$ and is given by

$$
\sum_{a: a \in \mathcal{A}_{i}^{+}} x_{a}^{k}-\sum_{a: a \in \mathcal{A}_{i}^{-}} x_{a}^{k}=d_{i}^{k} \quad \forall i \in \mathcal{N}, k \in \mathcal{D} .
$$

In terms of the standard node-arc incidence matrix $A$ of the network and the demand function $d^{k}(t)$, these constraints can be rewritten as

$$
A x^{k}=d^{k}(t) \quad \forall k \in \mathcal{D} .
$$

The second condition ensures that if there is positive flow of commodity $k$ along arc $a$, then the corresponding time to deliver that commodity is minimized:

$$
0 \leq c_{a}(f)+t_{j}^{k}-t_{i}^{k} \perp x_{a}^{k} \geq 0 \quad \forall a=(i, j) \in \mathcal{A}, k \in \mathcal{D},
$$

where the arc flow vector $f$ is given by

$$
f \equiv \sum_{k \in \mathcal{D}} x^{k} .
$$

This is typically termed "Wardrop's second principle," although it appears first in his article [205]. It is clear that given an enumeration of the paths, the solution generated from a path-based formulation can easily yield a solution to the multicommodity formulation, and vice versa.

Eliminating the flow vector $f$, conditions (3.34) and (3.35) defined a mixed NCP in the arc flow vector $x$ and minimum travel cost vector $t$. In what follows, we discuss some special cases of this general multicommodity equilibrium flow problem that are derived from particular cost functions $c_{a}(f)$ and demand functions $d^{k}(t)$.

Early studies of the traffic equilibrium problem have focused on the case of a separable cost function and constant demand function (see, e.g., [188]); that is, for all $a \in \mathcal{A}$ and $k \in \mathcal{D}$,

$$
c_{a}(f)=c_{a}\left(f_{a}\right) \quad \text { and } \quad d^{k}(t)=d^{k} \text { (a constant). }
$$

In this case, letting $C_{a}\left(f_{a}\right)$ be the integral of $c_{a}\left(f_{a}\right)$, we see that the equilibrium conditions (3.34) and (3.35) are the first-order optimality conditions of the nonlinear program

$$
\begin{array}{ll}
\operatorname{minimize} & \sum_{a \in \mathcal{A}} C_{a}\left(\sum_{k \in \mathcal{D}} x_{a}^{k}\right) \\
\text { subject to } & A x^{k}=d^{k}, x^{k} \geq 0 \quad \forall k \in \mathcal{D} .
\end{array}
$$

If each $C_{a}(\cdot)$ is a convex function, then this nonlinear program is a convex multicommodity network flow problem [110]. Recent algorithmic work has used parallel machines to exploit the network structure further. In many practical instances, there are upper bounds on the arc flow variables $f_{a}$ since the arcs have finite capacities; these bounds would impose additional constraints to the formulation (3.36).

A specific example of a separable cost function $c_{a}$ is given by

$$
c_{a}\left(f_{a}\right)=A_{a}+B_{a}\left[\frac{f_{a}}{\gamma_{a}}\right]^{4} \quad \forall a \in \mathcal{A}
$$


for particular data $A_{a}, B_{a}$, and $\gamma_{a}$. Cost functions of this kind have been used extensively in transportation research. Further details can be found in [46]. Nonseparable and nonintegrable cost functions $c(f)$ have also been used in the literature; see, for example, [54]. For such functions, the equilibrium conditions (3.34) and (3.35) do not correspond to the first-order optimality conditions of an optimization problem.

When the travel demand is not a constant, that is, when $d^{k}(t)$ depends on $t$, the optimization problem (3.36) is no longer a valid formulation of the multicommodity equilibrium flow problem defined by conditions (3.34) and (3.35), even if the cost function $c_{a}(f)$ is separable. However, if both $c_{a}(f)$ and $d^{k}(t)$ are separable for all $a \in \mathcal{A}$ and $k \in \mathcal{D}$, say

$$
d_{i}^{k}(t)=d_{i}^{k}\left(t_{i}^{k}\right) \quad \forall k \in \mathcal{D}, i \in \mathcal{N},
$$

and if $d_{i}^{k}\left(t_{i}^{k}\right)$ has an inverse function $t_{i}^{k}\left(d_{i}^{k}\right)$, then (3.34) and (3.35) are in fact the first-order optimality conditions of the following nonlinear program in the variables $(x, f, d)$ :

$$
\begin{array}{ll}
\operatorname{minimize} & \sum_{a \in \mathcal{A}} C_{a}\left(f_{a}\right)-\sum_{i \in \mathcal{N}, k \in \mathcal{D}} T_{i}^{k}\left(d_{i}^{k}\right) \\
\text { subject to } & A x^{k}-d^{k}=0, x^{k} \geq 0 \quad \forall k \in \mathcal{D}, \\
& f-\sum_{k \in \mathcal{D}} x^{k}=0
\end{array}
$$

where $C_{a}\left(f_{a}\right)$ and $T_{i}^{k}\left(d_{i}^{k}\right)$ are, respectively, the integral of $c_{a}\left(f_{a}\right)$ and $t_{i}^{k}\left(d_{i}^{k}\right)$.

An example of a separable demand function is

$$
d_{i}^{k}\left(t_{i}^{k}\right)=\bar{d}_{i}^{k} \frac{\exp \left(-\rho t_{i}^{k}\right)}{\exp \left(-\rho t_{i}^{k}\right)+\exp \left(-\rho \bar{t}_{i}^{k}\right)},
$$

where $\rho, \bar{d}_{i}^{k}$, and $\bar{t}_{i}^{k}$ are given constants. The inverse of this function is

$$
t_{i}^{k}\left(d_{i}^{k}\right)=\bar{t}_{i}^{k}+1 / \rho \ln \left(\frac{\bar{d}_{i}^{k}}{d_{i}^{k}}-1\right)
$$

for $0<d_{i}^{k}<\bar{d}_{i}^{k}$. Note the potential numerical difficulty when $d_{i}^{k}$ is close to the two bounds.

In summary, we see that the traffic equilibrium models are not always representable as nonlinear programs, since there are many instances where the arc cost and travel demand functions are not integrable or the inverse demand functions cannot be expressed in closed form. For more discussion of the complementarity approach to traffic equilibrium, see [70].

3.6.3. Network design problems. The traffic equilibrium problems described above have appeared as constraints of an MPEC in at least two instances of optimal network design. In [127], the arc cost function $c(f, \gamma)$ is assumed to depend on a vector of design variables $\gamma \in \mathbf{R}^{|\mathcal{A}|}$ (e.g., arc capacities). The first-level objective function is taken to be the sum of the investment cost $g(\gamma)$, where $g: \mathbf{R}^{|\mathcal{A}|} \rightarrow \mathbf{R}$ is given, and the system $\operatorname{cost} f^{T} c(f, \gamma)$. The overall design problem can be formulated as the following MPEC:

$$
\begin{array}{ll}
\text { minimize } & f^{T} c(f, \gamma)+g(\gamma) \\
\text { subject to } & \gamma \in \Gamma \\
\text { and } & \text { either [(3.32) and (3.33)] or (3.37), }
\end{array}
$$

where $\Gamma \subseteq \Re^{|\mathcal{A}|}$ is the constraint set of the arc capacities (e.g. a rectangular box). 
In [25], Chen and Florian have proposed a two-level optimization approach for the O-D demand adjustment problem. In what follows we shall use the multicommodity formulation of the traffic flow problem to describe this two-level optimization problem. The underlying setting is that a set of O-D demands $d_{\text {giv }}^{k}, k \in \mathcal{D}$ is available, and the network planner wishes to adjust these demands minimally in order to satisfy the equilibrium conditions. Using a least-squares measure of deviation, the MPEC formulation of this O-D demand adjustment problem is as follows:

$$
\begin{array}{ll}
\operatorname{minimize} & \sum_{(i, k) \in \mathcal{N} \times \mathcal{D}}\left(d_{i}^{k}-\left(d_{\text {giv }}^{k}\right)_{i}\right)^{2} \\
\text { subject to } & (3.36) .
\end{array}
$$

3.7. Optimal control problems. We briefly discuss a continuous-time optimal control problem whose discretization results in an ELQP; more details are given in [40]. A fixed interval $\left[t_{0}, t_{1}\right]$ is divided into $n$ segments over which control variables $u(t)$ and state variables $z(t)$ are discretized. Under this discretization, the variables $x=\left(u^{L}, u^{1}, \ldots, u^{n}, z^{1}, \ldots, z^{n}, z^{R}\right)$ and $y=\left(v^{1}, \ldots, v^{n}, v^{R}, w^{L}, w^{1}, \ldots, w^{n}\right)$ are constrained to lie in simple boxes $X$ and $Y$. Here, $v$ and $w$ correspond to dual control and state variables, respectively, and the subscripts $L$ and $R$ denote data and variables used to define boundary conditions at the left and right endpoints. The resulting linear quadratic function $\mathcal{L}$ is given by (2.4), where the matrices $P, p, Q, q$, and $R$ are given by

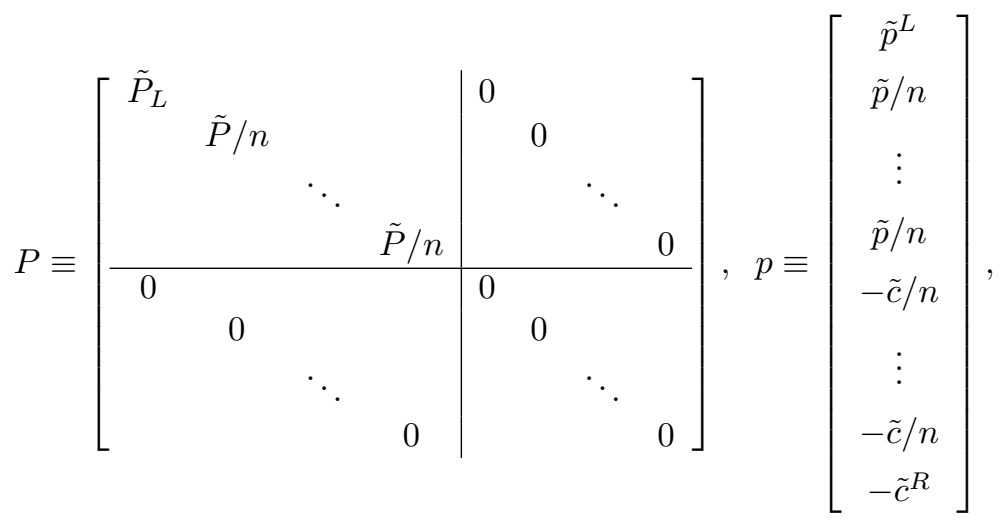

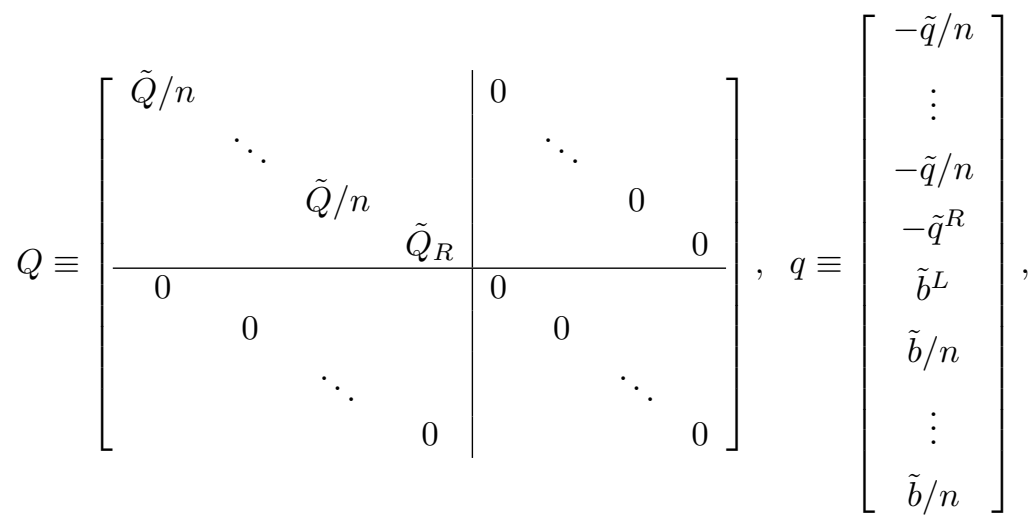


and

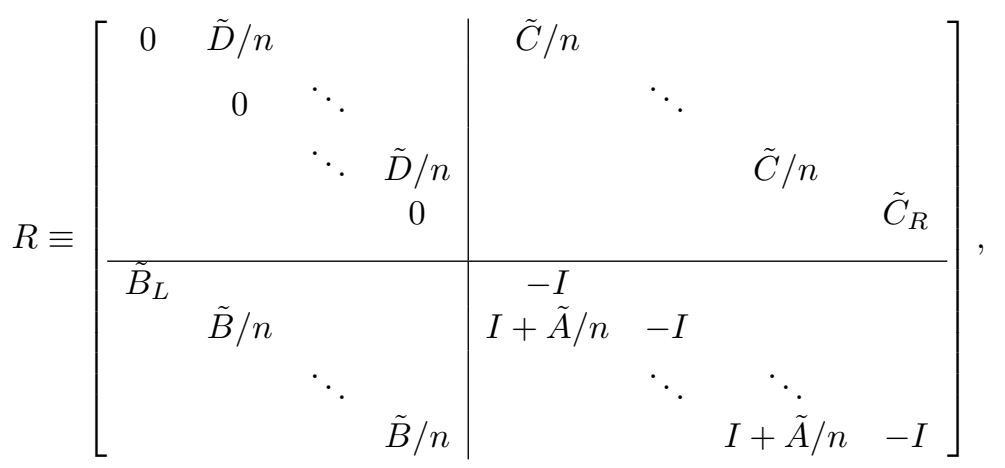

and the dots represent replication $n$ times. The tilde notation is used to signify data that arises in the continuous-time problem. The GAMS implementation described in [40] generates the data elements from the continuous-time problem randomly, with the matrices $P$ and $Q$ being positive semidefinite. While it is possible to express the discrete-time problem without using the state variables, their elimination results in a dense problem. For this reason, the state variables are retained in the GAMS formulation.

4. Economic applications. In many economic applications, a model is generated in order to gain insight into the complex workings of an economic system or to determine the effects of a certain action such as taxation. Different assumptions on the behavior of the agents in the economy determine different forms of equilibria.

An economy can be broadly modeled as follows. It is assumed that the economy deals in a finite number $\ell$ of commodities, which are determined not only by what type of good is being considered but also by date and location. Thus wheat in Chicago on Saturday may be distinguished from wheat in Chicago on Sunday and wheat in Minneapolis on Saturday. Associated with each commodity is a price, the amount paid now for every unit of the given commodity. There are two sets of economic agents dealing in these commodities: sectors (or firms or producers) and consumers. The wealth or budget of a consumer is typically determined from an initial endowment of the goods. In this description the economy is assumed to have $n$ sectors and $m$ consumers. Subscripts will be used to index elements of vectors and superscripts to index vector quantities associated either with the sector or the consumer. The case where there are no sectors of production is called a pure exchange economy.

Scarf [176] originally demonstrated the feasibility and potential of numerical modeling in the Arrow-Debreu general equilibrium framework using fixed-point methods. More recent work [129] has used pivotal techniques based on Lemke's algorithm [112]. The scope of economic questions has been extended to include public finance [10], international trade $[67,158,183]$ and development planning $[1,38,64]$. Other extensions will be noted in what follows.

4.1. General equilibrium. A general equilibrium problem (that is commonly attributed to Arrow-Debreu [7] and Walras [203]) is to find commodity prices, sector activities, and consumer consumption vectors such that

$$
\text { each sector maximizes its profit; }
$$

supply exceeds demand;

expenditure equals income. 
All these statements make intuitive sense. The first statement describes the behavior of the producers; namely, their goal is to maximize the firm's profit. The second statement is natural from the first; namely, if some commodity were in excess demand, then it would be profitable for some sector to produce that commodity, resulting in a supply increase until the demand becomes satisfied; the last statement is usually termed the Walras law; it stipulates that the total expenditure of the consumers is equal to the income generated by the trade of the commodities.

An underlying assumption is that there is perfect competition: consumers do not have a favorite producer, everyone is a price taker, and each consumer and sector has perfect information about these prices. Under specific forms of production and consumption, the above statements lead to a complementarity formulation of the economic model. Several examples are given below.

4.1.1. Production. The production side of an economy can be described using a production vector $y^{j} \in \mathbf{R}^{l}$ of each sector $j$. The components of this vector are positive if the sector produces more of the commodity than it consumes and are negative if the sector consumes more than it produces. Each sector is constrained by its technology, which is conceptually modeled using the production set $Y^{j} \subseteq \mathbf{R}^{\ell}$. It is assumed that $Y^{j}$ is a closed set and contains the vector 0 , so that zero production is possible. In a competitive marketplace, the $j$ th sector makes the profit $\pi_{j}(p)$ by solving the optimization problem

$$
\pi_{j}(p)=\max \left\{p^{T} y^{j}: y^{j} \in Y^{j}\right\} .
$$

It is assumed that sector $j$ is a price taker; that is, its actions do not affect the prices in the economy. This is a special case of general equilibrium: in this economy, prices are determined by technology alone; levels of production are determined by consumers.

The variables $y^{j}$ are sometimes referred to as netput vectors, since they give the net output of sector $j$. In many models, these netput vectors are determined from vectors of input and output commodities. Frequently, the production set $Y^{j}$ is specified by a functional relationship between these inputs and outputs and the activity level of the sector.

The standard example uses a technology matrix

$$
A=\left[\begin{array}{llll}
a^{1} & a^{2} & \cdots & a^{n}
\end{array}\right] \in \mathbf{R}^{\ell \times n},
$$

whose entries are typically a nonlinear function of the price vector $p$. Later, we will describe a widely used scheme to determine the technology matrix $A$ using production functions. The matrix $A$ converts levels of activity of producers $z \in \mathbf{R}^{n}$ into netput vectors of commodities. To see how this works, consider a linear activity analysis model $[61,103]$. The activity of sector $j, z_{j} \geq 0$ is converted into commodities $y^{j}$ by

$$
y^{j}=a^{j} z_{j} .
$$

Each sector must maximize profits at prices $p$, so (4.4) becomes

$$
\max \left\{p^{T} a^{j} z_{j}: z_{j} \geq 0\right\} .
$$

The necessary and sufficient optimality conditions for all the sectors are

$$
0 \leq z \perp A^{T} p \leq 0 .
$$

In essence this last condition stipulates the (nonnegativity and) complementarity between the two vectors $z$ and $A^{T} z$. 
We will now describe how the technology of the sectors is determined in a large class of economic models using production functions. These functions determine a scalar output level $f(x)$ from an input $x$. Normally an additional assumption regarding constant returns to scale for production is made. When a sector's production is modeled using a production function $f$, constant returns to scale means the production function is positively homogeneous of degree 1; that is, $f(\eta x)=\eta f(x)$ for all $\eta>0$. Typically, large numbers of sectors and consumers are modeled, so that any sector can be assumed to be small compared with the size of the market, making this a reasonable assumption.

The relation (4.1) can be replaced under constant returns to scale by the following statement:

$$
\text { each sector makes no excess profit. }
$$

This is due to the fact that if some sector were to make a positive profit, then by replicating its activity, the sector would make twice the profit, and thus its total profit would be unbounded. Intuitively, the mimicking behavior drives the price of the corresponding commodity down and hence reduces the profitability of that commodity. Of course, if the activity is unprofitable, then the corresponding activity of the sector is zero and therefore profit is zero.

The most widely used class of functions in the economics literature is the socalled CES (constant elasticity of substitution) functions. The general form of CES functions is

$$
\left(\sum_{i=1}^{l} \lambda_{i} x_{i}^{\rho}\right)^{1 / \rho}, \quad \lambda_{i} \geq 0, \quad i=1, \ldots, \ell .
$$

The domain of these functions is normally assumed to be $\mathbf{R}_{+}^{\ell}$, with the values on the boundaries defined by continuity. It can be shown that such functions are convex for $\rho \geq 1$ and concave for $\rho \leq 1$.

Notice the following special cases.

1. Letting $\rho=1$ gives the linear production function:

$$
\sum_{i=1}^{l} \lambda_{i} x_{i}
$$

2. When $\sum_{i=1}^{l} \lambda_{i}>0$, we can determine the limit as $\rho \downarrow 0$ using l'Hôpital's rule as

$$
\prod_{i=1}^{l} x_{i}^{\mu_{i}}
$$

where $\mu_{j} \equiv \lambda_{j} / \sum_{i=1}^{l} \lambda_{i}$. This is the Cobb-Douglas production function.

3. We can determine the limit as $\rho \rightarrow-\infty$ as

$$
\min _{1 \leq i \leq \ell} \lambda_{i} x_{i}
$$

This is the Leontief production function. Note that it is a concave function. Typically the parameters of the CES functions are calibrated using a reference point to match function and gradient information supplied by the modeler at a benchmark [179]. Furthermore, in many larger examples, CES functions are nested to several levels to model "local" interactions as well as "global" interactions [156]. 
Most of the practical models use one of these production functions to determine the technology matrix of the sector in the following manner. The netput vector $y^{j}$ of a given sector $j$ is split into its inputs $x$ and outputs $q$. Assuming the sector runs at an activity level $z_{j}$, the profit is given by

$$
\max _{x, q}\left\{p^{T}(q-x): f^{j}(x)=z_{j}, \quad g^{j}(q)=z_{j}, \quad x, q \geq 0\right\} .
$$

Here $f^{j}$ is a concave CES function and $g^{j}$ is a convex CES function that has $\rho \geq 1$ in (4.7). The latter is commonly termed a constant elasticities of transformation (CET) function. Note that the inputs and outputs are generated from an activity level $z_{j}$ using elasticities of substitution from these CES functions. Of course, $g^{j}$ can include the special case of a single output good. Since $f^{j}$ and $g^{j}$ are positively homogeneous of degree 1, the profit can be calculated as

$$
\pi_{j}(p)=p^{T} a^{j}(p) z_{j}
$$

where

$$
a^{j}(p) \equiv q^{j}-x^{j}
$$

and $\left(x^{j}, q^{j}\right)$ solve

$$
\max _{x, q}\left\{p^{T}(q-x): f^{j}(x)=1, \quad g^{j}(q)=1, \quad x, q \geq 0\right\} .
$$

The solution for this optimization problem is generally determined analytically to give an expression for $a^{j}(p)$. The conditional factor demands for unit output $x^{j}$ and the conditional outputs of the sector $q^{j}$ can be determined separately by solving

$$
\min _{x}\left\{p^{T} x: f^{j}(x)=1, x \geq 0\right\}
$$

and

$$
\max _{q}\left\{p^{T} q: g^{j}(q)=1, q \geq 0\right\}
$$

respectively. As a particular example, if $f$ is a Cobb-Douglas function, then

$$
x_{k}^{j}(p)=\frac{\lambda_{k} \prod_{j=1}^{\ell}\left(p_{j} / \lambda_{j}\right)^{\lambda_{j}}}{p_{k}}
$$

Note that as a function of $p, x^{j}$ is positively homogeneous of degree 0 . The optimal activity levels $z$ are then determined from (4.5). The supply from sector $j$ is $a^{j}(p) z_{j}$, giving total supply $A(p) z$. It remains to calculate the consumers demand, which is the subject of the next section.

4.1.2. Consumption. The consumption side of the economy is similarly defined. The consumption vector $d^{i} \in \mathbf{R}_{+}^{\ell}$ of each consumer $i$ indicates the quantity of the commodity that is consumed. The consumption set $X^{i} \subseteq \mathbf{R}_{+}^{\ell}$ models the minimal consumption constraints imposed on the $i$ th consumer. A key notion for consumers is the idea of preferences, that is, a complete preordering on the set of commodities. Thus a consumer may prefer a bundle of commodities $d$ to the bundle $e$ or may be indifferent between them or may prefer $e$ to $d$. Normally, this is represented by a utility function $u^{i}$, with the property that $u^{i}(d)>u^{i}(e), u^{i}(d)=u^{i}(e)$, or $u^{i}(d)<u^{i}(e)$, respectively. In most cases, $u$ is continuous and is generally assumed to be a CES, 
Cobb-Douglas, or Leontief function. Further, nonsatiation is normally assumed; that is, given any bundle of commodities $d^{i} \in X^{i}$, there is always another bundle $e^{i} \in X^{i}$ that the $i$ th consumer would prefer. The $i$ th consumer is endowed with an initial wealth $w_{i}$, and assuming that he is also a price taker, he will maximize his utility in the competitive paradigm, subject to his budget and consumption constraints. Thus, the $i$ th consumer would solve

$$
\max \left\{u^{i}\left(d^{i}\right): p^{T} d^{i} \leq w_{i}, d^{i} \in X^{i}\right\} .
$$

Under local nonsatiation (more precisely, the fact that with most utility functions, every commodity is desirable), the budget constraint will be binding at the optimal solution.

Typically, wealth is determined from an initial endowment of commodities $e^{i}$, so that $w_{i}=p^{T} e^{i}$. Unfortunately, in many models, $X^{i}=\mathbf{R}_{+}^{\ell}$, and thus (4.10) is not solvable when some $p_{i}=0$ and commodity $i$ is desirable. It is therefore assumed that $p>0$ and $X^{i}=\mathbf{R}_{+}^{\ell}$ in these modeling applications, although compactness of $X^{i}$ and the feasibility of (4.10) would remove the solvability difficulties when $p_{k}=0$ for one or more $k$. Under these assumptions, the demand of consumer $i$ depends on the utility function, prices, and endowment as follows. Using the Leontief utility,

$$
d_{k}^{i}\left(p, w_{i}\right)=\frac{w_{i}}{\lambda_{k} \sum_{j=1}^{\ell} p_{j} / \lambda_{j}} .
$$

For the case of Cobb-Douglas utility,

$$
d_{k}^{i}\left(p, w_{i}\right)=\frac{\lambda_{k} w_{i}}{p_{k}}
$$

whereas for CES utility,

$$
d_{k}^{i}\left(p, w_{i}\right)=\frac{\left(p_{k} / \lambda_{k}\right)^{r-1} w_{i}}{\sum_{j=1}^{\ell} p_{j}^{r} / \lambda_{j}^{r-1}}
$$

where $r=\rho /(\rho-1)$. Note that $d$ is positively homogeneous of degree 0 in all the above cases. Furthermore, when $w_{i}=p^{T} e^{i}$, then $d$, considered as a function of $p$ alone, is still positively homogeneous of degree 0 .

Summing all the information regarding production and consumption results in the following complementarity problem, we have

$$
\begin{gathered}
0 \leq z \perp-A(p)^{T} p \geq 0, \\
0 \leq p \perp A(p) z-\sum_{i=1}^{m}\left(d^{i}\left(p, w_{i}\right)-e^{i}\right) \geq 0, \\
w_{i}=p^{T} e^{i} .
\end{gathered}
$$

Here, $A(p)$ is determined by (4.8) and (4.9) as sectors maximizing profit, and $d^{i}\left(p, w_{i}\right)$ is determined by consumers maximizing utility using one of (4.11), (4.12), or (4.13). It is interesting to note that there is an enormous amount of skew symmetry in the above formulation that may be exploited by particular solution techniques.

If $(p, z, w)$ solves the above problem, then so does $(\lambda p, z, \lambda w)$ for any $\lambda>0$. This frequently causes algorithms difficulty in determining a solution. Several strategies have been considered in the literature. One fixes the price of a particular good, another fixes the sum of the prices, and yet another fixes an income level of one of 
the consumers. The first technique suffers from the fact that the fixed price may be zero, while the second increases the dimension of the problem and introduces an arbitrary column corresponding to the multiplier added for the extra constraint. Fixing the income of a consumer reduces the size of the problem without the danger of introducing inconsistencies into the model. Numerically, it is somewhat advantageous to leave the income constraints explicit as opposed to substituting the values into the demand function since the latter technique will generate much more dense Jacobian matrices.

Note that a more general formulation is possible with fewer explicit assumptions on the profit function $\pi(p)$. Two standard results are used to generate a similar formulation to that given above. The first is Hotelling's lemma [200] which states that if the profit function defined using (4.4) has a unique solution, then it is differentiable, and the optimal solution vector (that is, the netput supply vector) is $\nabla \pi(p)$. This solution can be substituted into (4.2). Frequently, the result is termed the envelope theorem. The complementarity problem that results from substituting this into (4.1) and (4.2) is

$$
\begin{gathered}
0 \leq z \perp-\pi(p) \geq 0, \\
0 \leq p \perp \nabla \pi(p) z-\sum_{i=1}^{m}\left(d^{i}\left(p, w_{i}\right)-e^{i}\right) \geq 0, \\
w_{i}=p^{T} e^{i} .
\end{gathered}
$$

Another result that enables the skew symmetry to be recaptured is Euler's law [200]. This states that if $\pi$ is a positively homogeneous function of degree 1 and is differentiable, then

$$
\pi(p)=\nabla \pi(p)^{T} p .
$$

Many of the remarks above apply per se to this formulation as well.

4.1.3. Taxation and subsidies. When taxes are applied to inputs or outputs, the profitability of the corresponding sectors and how the sectors technology is operated may be affected. Similar consequences occur with subsidies, but we note that questions regarding subsidies can be answered using negative tax rates.

It is clear that the conditional factor demands, that is, an $x$ which solves (4.9), and the conditional outputs of the sector $q$ can be determined separately. When input costs are distorted by taxes, sector $j$ solves the following problem:

$$
\min _{x}\left\{\sum_{i=1}^{l}\left(1+t_{i}\right) p_{i} x_{i}: f^{j}(x)=1, x \geq 0\right\},
$$

where $t_{i}$ is the tax rate on inputs. Similarly, if $\tau_{i}$ is the tax rate on outputs, then the sector determines its outputs $q$ based on

$$
\max _{q}\left\{\sum_{i=1}^{l}\left(1-\tau_{i}\right) p_{i} q_{i}: g^{j}(q)=1, q \geq 0\right\} .
$$

Supply is given by $A(p) z$ where the columns of $A$ are defined using (4.8) and the solutions of the above problems. However, the taxes affect the sector's profit. If we define

$$
C(p) \equiv\left[\begin{array}{llll}
c^{1} & c^{2} & \cdots & c^{n}
\end{array}\right]
$$


with $c_{i}^{j}(p)=q_{i}^{j}\left(1-\tau_{i}\right)-x_{i}^{j}\left(1+t_{i}\right)$, then the after-tax profit is given by $C(p)^{T} p$. Furthermore, tax revenue of $T \equiv(A(p)-C(p))^{T} p$ per unit activity level is accrued that is assumed to be shared among the consumers. Let $\theta_{j k}$ represent the share of sector $j$ taxes that accrue to consumer $k$.

The resulting set of equilibrium conditions are now given by

$$
\begin{gathered}
0 \leq z \perp-C(p)^{T} p \geq 0, \\
0 \leq p \perp A(p) z-\sum_{i=1}^{m}\left(d^{i}\left(p, w_{i}\right)-e^{i}\right) \geq 0, \\
w_{i}=p^{T} e^{i}+\sum_{j=1}^{n} \theta_{j k} T_{j} z_{j} .
\end{gathered}
$$

Taxation effects in a single country were explored in the GEMTAP model described in [10]. Similar models are currently being used to study taxation and other topics in public finance [74].

Exchange rates, linkages between domestic and international economies and intertemporal effects are currently being studied. The reader is referred to [173], which describes a modeling framework for such issues. Some work on increasing returns to scale can be found in [114]. Decreasing returns to scale can be modeled within the constant returns to scale framework using specific factors [173]. Other computable general equilibrium (CGE) models have considered the effect of carbon emission restrictions on international trade $[83,126,155]$.

Although the conventional first assumption in applied general equilibrium modeling starts with an assumption of perfect competition, there are instances that result in a monopoly or an oligopoly. An example of modeling with imperfect competition is given in [114].

4.2. Invariant capital stock. The problem of determining an invariant optimal capital stock is considered in [36, 68]. An economy is assumed to evolve over an infinite number of time periods with constant production and resources in each time period. At the beginning of each time period, the economy invests its capital goods $z_{t}$ into the production processes, which produce both capital goods and consumption goods $x_{t}$. The capital produced will be invested in the next period, while the consumption goods produced determine the utility of the investment. The total utility is a discounted sum; that is, the utility earned by consumption at time $t$ is discounted by a factor of $\alpha^{t}$, where the discount factor $\alpha \in(0,1)$. The problem can be formulated as

$$
\left.\begin{array}{ll}
\operatorname{maximize} & \sum_{t=0}^{\infty} \alpha^{t} u\left(x_{t}\right) \\
\text { subject to } & \\
& A x_{t} \leq z_{t} \\
& B x_{t} \geq z_{t+1} \\
& C x_{t} \leq w \\
& x_{t} \geq 0,
\end{array}\right\} \forall t=0,1, \ldots,
$$

where $u(x)$ is the utility derived from the consumption specified as $x$. The feasibility conditions ensure that the growth path $\left\{\left(z_{t}, x_{t}\right)\right\}$ determining these utilities is consistent with the given technology and resource constraints. $A$ is the capital input matrix; running production process $j$ at unit level requires $a_{i j}$ units of capital good $i$. $B$ is the capital output matrix; running production process $j$ at unit level produces $b_{i j}$ units of capital good $i$. $C$ is the resource input matrix; running production process $j$ 
at unit level requires $c_{r j}$ units of resource good $r$ of which we have $w$ available at the beginning of every time period.

Notice that in (4.14), the initial capital stock $z_{0}$ is given; this stock determines the optimal growth path. The sequence of capital stocks $\left\{z_{t}\right\}$ is not fixed explicitly by the constraints, but it is likely that, over time, some optimal pattern of investment and return may evolve; that is, the growth path approaches a constant value.

This motivates the problem of finding an initial endowment of capital $z_{0}$ for which the investment strategy necessary to maximize the discounted sum of the utilities is constant over all time periods. It should be noted that one cannot merely require that the path be constant, and optimize the choice of $z_{0}$. The invariance of the path must be a result of the optimality conditions in (4.14) and the choice of $z_{0}$, not of any explicit constraint.

We will assume that the utility function to be maximized in (4.14) is concave and continuously differentiable. The particular example given in [68] uses a utility function that is a nested CES function. The outer nest is Cobb-Douglas with decreasing returns to scale, while each of the inner nests is linear. Under some reasonable constraints on the technology, and a regularity condition on $z_{0}$, an initial capital stock $z_{0}$ yields a constant optimal growth path $\left(z_{t}, x_{t}\right)$ if and only if there exists $(x, y, v)$ solving the NCP

$$
\begin{aligned}
& 0 \leq x \quad \perp \quad-\nabla u(x)+(A-\alpha B)^{T} y+C^{T} v \geq 0, \\
& 0 \leq y \quad \perp \quad(B-A) x \geq 0, \\
& 0 \leq v \quad \perp \quad-C x+w \geq 0,
\end{aligned}
$$

and $x_{t}=x, z_{t}=A x$ for all $t$.

4.3. Game-theoretic models. A large number of economic models are formulated in terms of an $n$-person noncooperative game [136]. In the general setting, such a game consists of $n$ players, each of whom has an associated strategy set $X^{i} \subseteq \mathbf{R}^{n_{i}}$ and a utility function $u_{i}: X^{i} \rightarrow \mathbf{R}$. The utility of any given player depends on the strategies $x^{j}$ of all the players. When acting in a Nash manner, each player maximizes his own utility assuming other players' strategies are known. Thus for given strategies $z^{j}, j \neq i$, player $i$ solves the following optimization problem:

$$
\max \left\{u_{i}\left(z^{1}, \ldots, z^{i-1}, x^{i}, z^{i+1}, \ldots, z^{n}\right): x^{i} \in X^{i}\right\} .
$$

The vector $z=\left(z^{i}\right) \in X \equiv \prod_{i=1}^{n} X^{i}$ constitutes a Nash equilibrium for the game if for each $i=1, \ldots, n$,

$$
u_{i}\left(z^{1}, \ldots, z^{i-1}, x^{i}, z^{i+1}, \ldots, z^{n}\right) \leq u_{i}(z) \quad \forall x^{i} \in X^{i} .
$$

It is well known that the problem of computing a Nash equilibrium can be formulated as a variational inequality if each $X^{i}$ is a closed convex set and each function

$$
u_{i}\left(x^{1}, \ldots, x^{i-1}, \cdot, x^{i+1}, \ldots, x^{n}\right)
$$

is concave and differentiable in the $i$ th argument for each fixed

$$
\left(x^{1}, \ldots, x^{i-1}, x^{i+1}, \ldots, x^{n}\right) .
$$

Under these assumptions, by defining

$$
F(x) \equiv\left(\begin{array}{c}
-\nabla_{x^{1}} u_{1}(x) \\
\vdots \\
-\nabla_{x^{n}} u_{n}(x)
\end{array}\right) \quad \text { for } x=\left(x^{i}\right) \in X,
$$


a vector $z$ is a Nash equilibrium if and only if $z$ is a solution of the VI $(F, X)$. If in addition each $X^{i}$ is defined by a finite system of differentiable inequalities and equations satisfying appropriate constraint qualifications, then the latter VI is equivalent to a complementarity system via its KKT conditions.

In general, the concept of a Nash equilibrium is somewhat deficient. One deficiency is due to the possible existence of multiple equilibria as defined above, some of which Pareto dominate others. Determining which equilibrium is the desired "solution" of the game is generally not possible.

The Nash-Cournot production model is an application of the Nash equilibrium concept. This model concerns a number of firms, each producing a common good in a market. Each firm sets its production level so as to maximize its own profit, under the assumption that the production of the other firms remains constant. Intuitively, a Nash equilibrium is a production pattern in which no firm can increase its profit by unilaterally changing its level of production. Since no firm chooses to change its production, an equilibrium ensues. Here, equilibrium means that each firm does a best response given others' choices.

If we let $n$ denote the number of firms, $x_{i}$ the quantity of the good that firm $i$ produces, $\xi \equiv \sum_{i=1}^{n} x_{i}$ the total quantity being produced, then the problem relies on two functions of these variables. The first, the inverse demand function $p(\xi)$, gives the unit price at which consumers will demand (and actually purchase) a quantity $\xi$. The second, $C_{i}\left(x_{i}\right)$, is the production cost for firm $i$. In conformity with generally accepted economic behavior, the inverse demand function $p$ is assumed to be decreasing, the cost function $C_{i}$ to be convex, and the "industry revenue curve" $\xi p(\xi)$ to be concave in the (scalar) variable $\xi \geq 0$. Under these assumptions, the utility function of each firm

$$
x_{i} p\left(x_{i}+\sum_{j \neq i} x_{j}^{*}\right)-C_{i}\left(x_{i}\right)
$$

is concave in $x_{i}$ (see [134]) and the strategy set $X^{i}=\mathbf{R}_{+}$. The corresponding variational inequality is the nonlinear complementarity problem

$$
0 \leq x \perp F(x) \geq 0,
$$

where $F(x)=\left(F_{i}(x)\right)$ with

$$
F_{i}(x) \equiv C_{i}^{\prime}\left(x_{i}\right)-p\left(\sum_{i=1}^{n} x_{i}\right)-x_{i} p^{\prime}\left(\sum_{i=1}^{n} x_{i}\right) \quad \text { for } x \in \mathbf{R}_{+}^{n} \text { and } i=1, \ldots, n .
$$

The actual functions $p$ and $C$ used in [134] are defined below: for given parameters $c_{i}, L_{i}, \beta_{i}>0$, and $\gamma>1$,

$$
\begin{gathered}
p(\xi)=5000^{\frac{1}{\gamma}} \xi^{\frac{-1}{\gamma}} \\
C_{i}\left(x_{i}\right)=c_{i} x_{i}+\frac{\beta_{i}}{1+\beta_{i}} L_{i}^{\frac{1}{\beta_{i}}} x_{i}^{\frac{\beta_{i}+1}{\beta_{i}}} .
\end{gathered}
$$

An application of Nash equilibrium in marketing is found in [26]. The variables of this problem are prices; it is assumed that a consumer demand function dictates the quantities consumed at a given price. As opposed to the general equilibrium where the firms choose a quantity to produce, here they choose the price for their 
single output based on maximizing their profit in the Nash manner. This is called the Bertrand model in the literature. Larger models are found in [128]. An extension of this framework is given by von Stackelberg [184]; here it is assumed that one firm is the leader and hence knows that all the other firms will act in a Bertrand fashion. The leader can optimize his quantities using this information to obtain a different solution. This alternate analysis is not known to be a complementarity problem but is an example of an MPEC; references on the Stackelberg game problem include $[142,143,178,196]$.

In the game theory literature, algorithms for finding equilibria in two-person noncooperative games are well known; see [113]. We now describe an $n$ player extension of bimatrix games. Suppose the players are indexed as $j \in \mathcal{N} \equiv\{1,2, \ldots, n\}$. For simplicity, we will assume that every player has a choice of pure strategy from the set $\mathcal{P}=\{1,2, \ldots, m\}$. A "strategy profile" $s$ is an $n$ vector whose $j$ th element is an element of $\mathcal{P}$ corresponding to the pure strategy assigned to player $j$. The payoff to player $j$ arising from strategy profile $s$ is $A_{j s}$. In the game, player $j$ chooses a probability vector $p_{i}^{j}$ representing his probability of playing pure strategy $i$ in the equilibrium. Clearly,

$$
p_{i}^{j} \geq 0 \quad \forall i \in \mathcal{P}, j \in \mathcal{N}, \quad \sum_{i \in \mathcal{P}} p_{i}^{j}=1 \quad \forall j \in \mathcal{N} .
$$

We now introduce some further notation. Let $p^{-j}$ represent the $(n-1) \times m$ vector $\left(p^{1}, \ldots, p^{j-1}, p^{j+1}, \ldots, p^{n}\right)$ and $i(j, s)$ denote the pure strategy assigned to player $j$ in the strategy profile $s$. The expected payoff to player $j$ is given by

$$
u_{j}(p) \equiv \sum_{s} A_{j s} p(s)
$$

where $p(s)$, the probability of choosing strategy profile $s$, can be calculated using

$$
p(s)=\prod_{k \in \mathcal{N}} p_{i(k, s)}^{k} .
$$

Since the game is finite, the summation in the expectation can be rearranged so that

$$
u_{j}(p)=\sum_{i \in \mathcal{P}} p_{i}^{j} x_{i}^{j}\left(p^{-j}\right)
$$

where

$$
x_{i}^{j}\left(p^{-j}\right) \equiv \sum_{s: i(j, s)=i} A_{j s} \prod_{k \in \mathcal{N} \backslash\{j\}} p_{i(k, s)}^{k} .
$$

The nonlinear function $x_{i}^{j}$ represents the expected payoff to player $j$ using pure strategy $i$ under the assumption that the other players choose strategies given by $p^{-j}$. The strategy sets are just unit simplices, $X^{j}=\left\{p \in \mathbf{R}^{m}: p \geq 0, \sum_{i} p_{i}=1\right\}$. The resulting Nash equilibrium is the problem of finding $\bar{p}=\left(\bar{p}^{1}, \ldots, \bar{p}^{n}\right)$ such that for $j \in \mathcal{N}$,

$$
\bar{p}^{j} \in X^{j}, \quad \sum_{i \in \mathcal{P}} p_{i}^{j} x_{i}^{j}\left(\bar{p}^{-j}\right) \leq \sum_{i \in \mathcal{P}} \bar{p}_{i}^{j} x_{i}^{j}\left(\bar{p}^{-j}\right) \quad \forall p^{j} \in X^{j} .
$$

It is clear that this is just VI $\left(F, \prod_{j \in \mathcal{N}} X^{j}\right)$ with $F_{i}^{j}(p) \equiv-x_{i}^{j}\left(p^{-j}\right)$. The equivalence given in (2.1) allows this problem to be rewritten as a complementarity problem, where the matrices $A$ and $b$ are chosen to represent the constraints $\sum_{i \in \mathcal{P}} p_{i}^{j}=1$. 


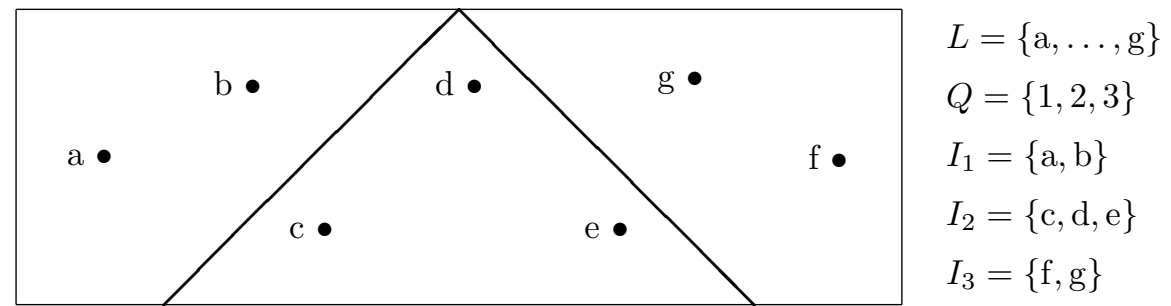

FIG. 4.1. Eight sites partitioned among three producers.

4.4. Spatial price equilibria. Some models are based on the spatial structure as well as the competitive structure of the market and are frequently termed spatial price equilibria. Examples of this format can be found in $[69,195]$. Some of these models are based on the general equilibrium framework; others use the Nash equilibrium framework.

In [69], Harker gives a number of models which describe the spatial and competitive structure of markets embedded in a network (such as those described in subsection 3.6). Each node represents a unit or site separated spatially from the others. One competitive structure modeled is an oligopoly, a market situation in which a few producers (or firms) control the deliveries to and demands from a large number of buyers. In the example below, each firm $q \in Q$ tries to maximize the profit associated with his production of a single commodity common to all firms. We define the following quantities (see Figure 4.1).

$[L]$ set of distinct production units or sites

$[W \subseteq L \times L]$ set of transportation arcs between the sites in $L$

$\left[I_{q} \subseteq L\right]$ set of sites controlled by firm $q \in Q$. The set of sites $L$ is partitioned among the sets $I_{q}, q \in Q$.

$\left[s_{l}, l \in L\right]$ amount of commodity produced at site $l$

$\left[C_{l}\left(s_{l}\right)\right]$ total cost of producing $s_{l}$ units of output at site $l$

$\left[f_{a}, a \in W\right]$ flow on $\operatorname{arc} a$

$\left[c_{a}\left(f_{a}\right), a \in W\right]$ unit transportation cost at level $f_{a}$

$\left[d_{l q}\right]$ amount of commodity produced by firm $q$ delivered to site $l$

$\left[\theta_{l}\left(\sum_{q \in Q} d_{l q}\right)\right]$ purchase price dictated by the total delivery to site $l$ (inverse demand function).

We will assume that each firm $q$ acts in a Nash manner when making decisions regarding $s, d$, and $f$. The aggregation of these variables is firm $q$ 's strategy vector $x^{q}$. The constraints on $x^{q}$ are those that ensure a conservation of flow at each site. Constraints for sites that firm $q$ controls are more complicated than those for sites outside of firm q's control. The supply, delivery, and transportation variables are subject to lower and upper bounds, taken as 0 and $+\infty$, respectively. Thus, the set $X^{q}$ of feasible strategies for the firm $q$ is

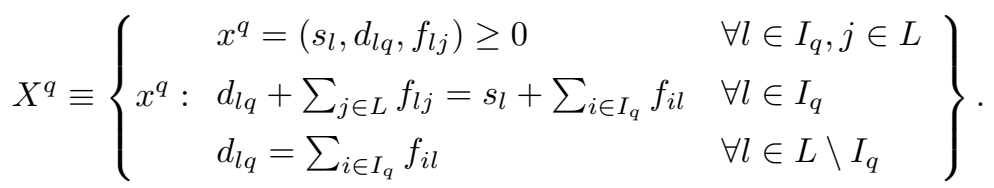

Let $X \equiv \prod_{q \in Q} X^{q}$, so that $x \in X$ is a feasible strategy for all firms. Firm $q$ 's profit 
is given by the function $\pi_{q}$ :

$$
\pi_{q}(x) \equiv \sum_{l \in L} \theta_{l}\left(\sum_{j \in L} f_{j l}\right) d_{l q}-\sum_{i \in I_{q}} C_{i}\left(s_{i}\right)-\sum_{i \in I_{q}} \sum_{j \in L} c_{i j}\left(f_{i j}\right) f_{i j} .
$$

Therefore, firm $q$ wishes to find a strategy $x^{q}$ which maximizes $\pi_{q}(x)$ under the assumption that all the other firms keep their strategies fixed at $\bar{x}^{p}$. If $\theta_{l}$ is decreasing, $C_{l}$ is convex, and $c_{i j}$ is increasing, then $\pi_{q}$ is convex. As in the Nash model, a spatial price equilibrium is therefore a vector $\bar{x} \in X$ satisfying

$$
\sum_{q \in Q} \nabla \pi_{q}(\bar{x})^{T}\left(x^{q}-\bar{x}^{q}\right) \geq 0 \quad \forall x \in X
$$

This variational inequality is easily transformed into a complementarity problem using (2.1). A GAMS model for this problem is available in [40].

In [195], Tobin describes a spatial price equilibrium in a multicommodity market modeled as a network. In this example, the variables are the prices at the various nodes in the network. These prices determine supply and demand, and not conversely, as in Harker's model above. Furthermore, perfect competition is assumed, rather than Nash behavior.

4.5. NEMS. NEMS stands for National Energy Modeling System. This is a very complex, large-scale mathematical model created at the U.S. Department of Energy that computes equilibrium fuel prices and quantities in the U.S. energy sector. The model consists of many modules that are described by linear programs, nonlinear equations, and econometric submodels which are linked together via some functional equations.

Currently, the integrating module within NEMS performs the role of a Walrasian auctioneer in the determination of equilibrium prices. It recursively invokes the independent submodels in a systematic fashion to obtain equilibrium market prices for energy products. Essentially, the algorithm used is a Gauss-Seidel procedure with some heuristics to improve robustness.

In a proposed complementarity formulation of NEMS [57], the conversion, transmission, and distribution of energy are modeled by four separate nonlinear programs. The mixed nonlinear complementarity problem is formed by augmenting the KKT conditions of these nonlinear programs with various nonlinear equations that link the solution vectors and multipliers to computed fuel prices and quantities.

5. Concluding remarks. We have given a fairly comprehensive summary of numerous applications of complementarity problems. Although many of these applications are known to researchers in the field, several of them have received only minimal attention in the complementarity literature. Indeed, we have recently learned about an interesting application of the linear complementarity problem arising from the financial field of option pricing [206, Chapter 7]. Further applications of the complementarity problem and related problems as well as recent algorithmic advances for solving these problems can be found in the Proceedings of the International Conference on Complementarity Problems held in November, 1995 [47]. Our hope with all these efforts is to stimulate further research and utilization of the complementarity approach to solving important engineering and economic problems.

Acknowledgments. We wish to thank several colleagues for offering constructive comments on the applications discussed in this survey and for sending us preprints 
of their work. Specifically, Professors Anders Klarbring and Francis Tin-Loi have helped us with the contact and structural mechanics problems, respectively; Professors Mark Cronshaw and Thomas Rutherford have commented on the general equilibrium section; Dr. Steven Gabriel and Professor Francis Tin-Loi have kindly sent us their preprints of $[57,192,194]$.

\section{REFERENCES}

[1] I. Adelman and S. Robinson, Income Distribution Policy in Developing Countries, Stanford University Press, Stanford, CA, 1978.

[2] A. M. Al-Fahed, G. E. Stavroulakis, and P. D. Panagiotopoulos, Hard and soft fingered robot grippers. The linear complementarity approach, Z. Angew. Math. Mech., 71 (1991), pp. 257-265.

[3] A. M. Al-Fahed, G. E. Stavroulakis, and P. D. Panagiotopoulos, A linear complementarity approach to the frictionless gripper, Internat. J. Robotic Research, 11 (1992), pp. $112-122$.

[4] P. Alart And A. Curnier, A mixed formulation for frictional contact problems prone to Newton like solution methods, Comput. Methods Appl. Mech. Engrg., 92 (1991), pp. 353-375.

[5] E. ANDERSEN AND Y. Ye, On a homogeneous algorithm for a monotone complementarity problem with nonlinear equality constraints, in [47], pp. 1-11.

[6] M. Anitescu And F. A. Potra, Formulating Dynamic Multi-Rigid-Body Contact Problems with Friction as Solvable Linear Complementarity Problems, Report 93, Department of Mathematics, The University of Iowa, Iowa City, IA, 1996.

[7] K. Arrow And G. Debreu, Existence of equilibrium for a competitive economy, Econometrica, 22 (1954), pp. 265-290.

[8] A. Auslender, Convergence of stationary sequences for variational inequalities with maximal monotone operators, Appl. Math. Optim., 28 (1993), pp. 161-172.

[9] A. Auslender and M. Haddou, An interior-proximal method for convex linearly constrained problems and its extension to variational inequalities, Math. Programming, 71 (1995), pp. $77-100$.

[10] C. Ballard, D. Fullerton, J. B. Shoven, and J. Whalley, A General Equilibrium Model for Tax Policy Evaluation, National Bureau of Economic Research Monograph, The University of Chicago Press, 1985.

[11] D. BARAFF, Issues in computing contact forces for nonpenetrating rigid bodies, Algorithmica, 10 (1993), pp. 292-352.

[12] D. BARAFF, Fast contact force computation for nonpenetrating rigid bodies, Computer Graphics (Proceedings SIGRAPH), 28 (1994), pp. 23-34.

[13] S. C. Billups, S. P. Dirkse, And M. C. Ferris, A comparison of large scale mixed complementarity problem solvers, Comput. Optim. Appl., 7 (1997), pp. 3-25.

[14] S. C. BIllups AND M. C. FerRIS, Convergence of an infeasible interior-point algorithm from arbitrary positive starting points, SIAM J. Optim., 6 (1996), pp. 316-325.

[15] S. C. Billups AND M. C. FerRis, QPCOMP: A quadratic program based solver for mixed complementarity problems, Math. Programming, 76 (1997), pp. 533-562.

[16] G. BJÖRKMAN, The solution of large displacement frictionless contact problems using a sequence of linear complementarity problems, Internat. J. Numer. Methods Engrg., 31 (1991), pp. 1553-1566.

[17] G. BJÖRKman, Path following and critical points for contact problems, Comput. Mech., 10 (1992), pp. 231-246.

[18] G. Björkman, A. Klarbring, T. Larsson, M. Rönnqvist, and B. Sjödin, Sequential quadratic programming for non-linear elastic contact problems, in Structural Optimization 93, The World Congress on Optimal Design of Structural Systems, J. Herskovits, ed., Vol. 2, 1993, pp. 301-308.

[19] A. Brooke, D. Kendrick, and A. Meeraus, GAMS: A User's Guide, The Scientific Press, South San Francisco, CA, 1988.

[20] D. Chan And J. S. PAng, The generalized quasi-variational inequality problem, Math. Oper. Res., 7 (1982), pp. 211-222.

[21] B. Chen AND P. T. HARKer, A noninterior continuation method for linear complementarity problems, SIAM J. Matrix Anal. Appl., 14 (1993), pp. 1168-1190. 
[22] B. Chen And P. T. Harker, A continuation method for monotone variational inequalities, Math. Programming, 69 (1995), pp. 237-253.

[23] C. Chen and O. L. Mangasarian, Smoothing methods for convex inequalities and linear complementarity problems, Math. Programming, 78 (1995), pp. 51-70.

[24] C. Chen And O. L. Mangasarian, A class of smoothing functions for nonlinear and mixed complementarity problems, Comput. Optim. Appl., 5 (1996), pp. 97-138.

[25] Y. Chen and M. Florian, O-D Demand Adjustment Problem with Congestion: Part I. Model Analysis and Optimality Conditions, Publication CRT-94-56, Centre de Recherche sur les Transports, Université de Montréal, Montréal, Canada, 1994.

[26] S. C. Choi, W. S. DeSarbo, And P. T. Harker, Product positioning under price competition, Management Science, 36 (1990), pp. 175-199.

[27] C. Cinquini and R. Contro, Optimal design of beams discretized by elastic plastic finite element, Comput. \& Structures, 20 (1985), pp. 475-585.

[28] C. Comi AND G. MAIER, Extremum theorem and convergence criterion for an iterative solution to the finite-step problem in elastoplasticity with mixed nonlinear hardening, European J. Mech. A Solids, 9 (1990), pp. 563-585.

[29] A. R. Conn, N. I. M. Gould, AND P. L. TOINT, Numerical experiments with the LANCELOT package (release A) for large-scale nonlinear optimization, Math. Programming, 75 (1996), pp. 73-110.

[30] T. F. Conry And A. SeIREg, A mathematical programming method for design for elastic bodies in contact, J. Appl. Mech., 38 (1971), pp. 1293-1307.

[31] R. W. Cottle And G. B. Dantzig, A generalization of the linear complementarity problem, J. Combin. Theory, 8 (1970), pp. 79-90.

[32] R. W. Cottle and M. S. Goheen, A special class of large quadratic programs, in Nonlinear Programming, Vol. 3, O. L. Mangasarian, R. R. Meyer, and S. M. Robinson, eds., Academic Press, London, 1978, pp. 361-390.

[33] R. W. Cottle, G. H. Golub, and R. S. Sacher, On the solution of large structured complementarity problems: The block partitioned case, Appl. Math. Optim., 4 (1978), pp. 347-363.

[34] R. W. Cottle, J. S. Pang, and R. E. Stone, The Linear Complementarity Problem, Academic Press, Boston, 1992.

[35] C. W. CRYER, The method of Christopherson for solving free boundary problems for infinite journal bearings by means of finite differences, Math. Comput., 25 (1971), pp. 435-553.

[36] G. B. Dantzig And A. S. Manne, A complementarity algorithm for an optimal capital path with invariant proportions, J. Econom. Theory, 9 (1974), pp. 312-323.

[37] O. De Donato And G. Maier, Mathematical programming methods for the inelastic analysis of reinforced concrete frames allowing for limited rotation capacity, Internat. J. Numer. Methods Engrg., 4 (1972), pp. 307-329.

[38] K. Dervis, J. De Melo, And S. Robinson, General Equilibrium Models of Development Policy, Cambridge University Press, Cambridge, England, 1982.

[39] S. P. Dirkse, Robust Solution of Mixed Complementarity Problems, Ph.D. thesis, Computer Sciences Department, University of Wisconsin, Madison, WI, 1994; also available from $\mathrm{ftp}: / /$ ftp.cs.wisc.edu/math-prog/tech-reports/.

[40] S. P. Dirkse And M. C. Ferris, MCPLIB: A collection of nonlinear mixed complementarity problems, Optimization Methods and Software, 5 (1995), pp. 319-345.

[41] S. P. Dirkse AND M. C. Ferris, The PATH solver: A non-monotone stabilization scheme for mixed complementarity problems, Optimization Methods and Software, 5 (1995), pp. 123-156.

[42] S. P. Dirkse AND M. C. FerRis, A pathsearch damped Newton method for computing general equilibria, Ann. Oper. Res., 68 (1996).

[43] S. P. Dirkse, M. C. Ferris, P. V. Preckel, and T. Rutherford, The GAMS callable program library for variational and complementarity solvers, Mathematical Programming Technical Report 94-07, Computer Sciences Department, University of Wisconsin, Madison, WI, 1994.

[44] A. A. Ebiefung And M. M. Kostreva, Global solvability of generalized linear complementarity problems and a related class of polynomial complementarity problems, in Recent Advances in Global Optimization, Princeton University Press, Princeton, NJ, 1991, pp. $102-124$.

[45] J. ECKSTEIN AND M. C. FeRRIS, Operator splitting methods for monotone affine variational inequalities, with a parallel application to optimal control, INFORMS J. Comput., (1997).

[46] M. C. Ferris, A. Meeraus, And T. F. Rutherford, Computing Wardropian equilibrium in a complementarity framework, Mathematical Programming Technical Report 95-03, Computer Sciences Department, University of Wisconsin, Madison, WI, 1995. 
[47] M. C. Ferris And J. S. PAng, eds., Complementarity and Variational Problems: State of the Art, SIAM, Philadelphia, PA, 1997.

[48] M. C. FERRIS AND D. RAlPh, Projected gradient methods for nonlinear complementarity problems via normal maps, in Recent Advances in Nonsmooth Optimization, D. Du, L. Qi, and R. Womersley, eds., World Scientific Publishers, River Edge, NJ, 1995, pp. $57-87$.

[49] M. Florian, ED., Traffic Equilibrium Methods, Springer-Verlag, Berlin, 1976.

[50] M. Florian, ED., Nonlinear cost network models in transportation analysis, Math. Programming Study, 26 (1986), pp. 167-196.

[51] R. Fourer, D. Gay, and B. Kernighan, AMPL, The Scientific Press, South San Francisco, CA, 1993.

[52] V. M. FRIEDMAN AND V. S. CherninA, Iterative methods applied to the solution of contact problems between bodies, Mekh. Tverd. Tela, 1 (1967), pp. 116-120 (in Russian).

[53] T. L. Friesz, Network equilibrium, design and aggregation, Transport. Res., 19A (1985), pp. $413-427$.

[54] T. L. Friesz, R. L. Tobin, T. E. Smith, and P. T. Harker, A nonlinear complementarity formulation and solution procedure for the general derived demand network equilibrium problem, J. Regional Sci., 23 (1983), pp. 337-359.

[55] M. Fukushima, Equivalent differentiable optimization problems and descent methods for asymmetric variational inequality problems, Math. Programming, 53 (1992), pp. 99-110.

[56] S. A. Gabriel And D. Berstein, A path generation method for the nonadditive, asymmetric, elastic traffic equilibrium problem, 1995, manuscript.

[57] S. A. Gabriel AND A. S. Kydes, A nonlinear complementarity approach for solving the national energy modeling system, Preprint MCS-P504-0395, Mathematics and Computer Science Division, Argonne National Laboratory, Argonne, IL, Mar. 1995.

[58] S. A. Gabriel And J. J. Moré, Smoothing of mixed complementarity problems, in Complementarity and Variational Problems: State of the Art, Philadelphia, PA, 1997.

[59] S. A. Gabriel AND J. S. PAng, An inexact NE/SQP method for solving the nonlinear complementarity problem, Comput. Optim. Appl., 1 (1992), pp. 67-91.

[60] S. A. Gabriel AND J. S. PANG, A trust region method for constrained nonsmooth equations, in Large-Scale Optimization: State of the Art, W. W. Hager, D. W. Hearn, and P. M. Pardalos, eds., Kluwer Academic Publishers, Norwell, MA, 1994, pp. 159-186.

[61] D. Gale, The Theory of Linear Economic Models, McGraw-Hill Book Company, New York, 1960

[62] Y. GAO, Nonlinear complementarity problems in large deformed beam subject to unilateral conditions, Department of Mathematics, Virginia Polytechnic Institute and State University, manuscript, Oct. 1994.

[63] C. B. Garcia And W. I. Zangwill, Pathways to Solutions, Fixed Points, and Equilibria, Prentice-Hall, Englewood Cliffs, NJ, 1981.

[64] V. Ginsburgh And J. Waelbroeck, Activity Analysis and General Equilibrium Modelling, North-Holland, Amsterdam, 1981.

[65] M. S. GOWDA AND R. SzNAJDER, The generalized order linear complementarity problem, SIAM J. Matrix Anal. Appl., 15 (1994), pp. 779-796.

[66] O. GÜLER, Existence of interior points and interior paths in nonlinear monotone complementarity problems, Math. Oper. Res., 18 (1993), pp. 128-147.

[67] J. HaAland, V. Norman, T. Rutherford, and T. Wergeland, VEMOD: A RicardoHeckscher-Ohlin-Jones model of world trade, Scandinavian Journal of Economics, (1987).

[68] T. Hansen And T. C. Koopmans, On the definition and computation of a capital stock invariant under optimization, J. Economic Theory, 5 (1972), pp. 487-523.

[69] P. T. HARKer, Alternative models of spatial competition, Oper. Res., 34 (1986), pp. 410-425.

[70] P. T. HARKER, Lectures on Computation of Equilibria with Equation-Based Methods, CORE Lecture Series, CORE Foundation, Louvain-la-Neuve, Université Catholique de Louvain, 1993.

[71] P. T. HARKer AND J. S. PANG, Existence of optimal solutions to mathematical programs with equilibrium constraints, Oper. Res. Lett., 7 (1988), pp. 61-64.

[72] P. T. HARker AND J. S. PANG, Finite-dimensional variational inequality and nonlinear complementarity problems: A survey of theory, algorithms and applications, Math. Programming, 48 (1990), pp. 161-220.

[73] P. T. HARKER AND B. XIAO, Newton's method for the nonlinear complementarity problem: A B-differentiable equation approach, Math. Programming, 48 (1990), pp. 339-358.

[74] G. W. HARrison, T. F. Rutherford, AND D. G. TARR, Trade reform in the partially liberalized economy of Turkey, The World Bank Economic Review, 7 (1993), pp. 191-217. 
[75] A. Haurie And P. Marcotte, On the relationship between Nash-Cournot and Wardrop equilibria, Networks, 15 (1985), pp. 295-308.

[76] D. W. Hearn, S. Lawphongpanich, and J. A. Ventura, Restricted simplicial decomposition: Computation and extensions, Math. Programming Study, 31 (1987), pp. 99-118.

[77] R. H. W. Hoppe And H. D. Mittelmann, A multi-grid continuation strategy for parameterdependent variational inequalities, J. Comput. Appl. Math., 26 (1989), pp. 35-46.

[78] Y. Hu, H. S. Cheng, T. Arai, Y. Kobayashi, and S. Aoyama, Numerical simulation of piston ring in mixed lubrication - a nonaxisymmetrical analysis, Trans. ASME, 116 (1994), pp. 470-478.

[79] G. IsAc, Complementarity Problems, Lecture Notes in Mathematics, Springer-Verlag, New York, 1992.

[80] G. IsAC And M. M. Kostreva, The generalized order complementarity problem, J. Optim. Theory Appl., 19 (1991), pp. 227-232.

[81] B. Jansen, K. Roos, T. Terlaky, And A. Yoshise, Polynomiality of affine-dual affinescaling algorithms for nonlinear complementarity problems, Discussion Paper 648, Institute of Socio-Economic Planning, University of Tsukuba, Ibaraki, Japan, 1995.

[82] L. JohansSON AND A. Klarbring, The rigid punch problem with friction using variational inequalities and linear complementarity, Mech. Structures Mach., 20 (1992), pp. 293-319.

[83] D. W. Jorgenson And P. J. Wilcoxen, Reducing US carbon emissions: An econometric general equilibrium assessment, Resource and Energy Economics, 15 (1993), pp. 7-25.

[84] N. H. Josephy, Newton's Method for Generalized Equations, Technical Summary Report 1965, Mathematics Research Center, University of Wisconsin, Madison, WI, 1979.

[85] I. KANEKO, A mathematical programming method for the inelastic analysis of reinforced concrete frames, Internat. J. Numer. Methods Engrg., 11 (1977), pp. 1137-1154.

[86] I. Kaneko, Piecewise linear elastic-plastic analysis, Internat. J. Numer. Methods Engrg., 14 (1979), pp. 757-767.

[87] I. KaneKo, Complete solution of a class of elastic-plastic structures, Comput. Methods Appl. Mech. Engrg., 21 (1980), pp. 193-209.

[88] C. KAnzow, Some equation-based methods for the nonlinear complementarity problem, Optimization Methods and Software, 3 (1994), pp. 327-340.

[89] C. Kanzow, Some Tools Allowing Interior-Point Methods to Become Noninterior, Tech. rep., Institute of Applied Mathematics, University of Hamburg, Germany, 1994.

[90] A. Klarbring, A mathematical programming approach to three dimensional contact problems with friction, Comput. Methods Appl. Mech. Engrg., 58 (1986), pp. 175-200.

[91] A. Klarbring, A quadratic program in frictionless contact problems, Intern. J. Engrg. Sci., 24 (1986), pp. 1207-1217.

[92] A. KLARBRING, On discrete and discretized non-linear elastic structures in unilateral contact (stability, uniqueness and variational principles), Internat. J. Solids and Structures, 24 (1988), pp. 459-479.

[93] A. Klarbring, Mathematical programming and augmented Lagrangian methods for frictional contact problems, in Proceedings Contact Mechanics International Symposium, A. Curnier, ed., Presse Polytechniques et Universitaires Romandes, Lausanne, 1992, pp. 409-422.

[94] A. Klarbring, Mathematical programming in contact problems, in Computational Methods for Contact Problems, Computational Mechanics Publications, Southampton, 1993, pp. 233-264.

[95] A. Klarbring, Large Displacement Frictional Contact: A Continuum Framework for Finite Element Discretization, Tech. rep. LiTH-IKP-R-798, Department of Mechanical Engineering, Linköping Institute of Technology, Linköping, 1994.

[96] A. Klarbring And G. BJÖRKman, A mathematical programming approach to contact problems with friction and varying contact surface, Comput. \& Structures, 30 (1988), pp. 1185-1198.

[97] A. Klarbring And G. BJÖrkman, Solution of large displacement contact problems with friction using Newton's method for generalized equations, Internat. J. Numer. Methods Engrg., 34 (1992), pp. 249-269.

[98] A. Klarbring And J. S. PAng, Existence of solution to discrete semicoercive frictional contact problems, SIAM J. Optim., to appear.

[99] M. Kojima, N. Megiddo, And T. Noma, Homotopy continuation methods for nonlinear complementarity problems, Math. Oper. Res., 16 (1991), pp. 754-774.

[100] M. Kojima, N. Megiddo, T. Noma, And A. Yoshise, A Unified Approach to Interior Point Algorithms for Linear Complementarity Problems, Lecture Notes in Comput. Sci. 538, Springer-Verlag, Berlin, 1991. 
[101] M. Kojima, S. Mizuno, And T. Noma, A new continuation method for complementarity problems with uniform P-function, Math. Programming, 43 (1989), pp. 107-113.

[102] M. Kojima, T. Noma, And A. Yoshise, Global convergence in infeasible interior-point algorithms, Math. Programming, 65 (1994), pp. 43-72.

[103] T. C. Koopmans, Ed., Activity Analysis of Production and Allocation, John Wiley, New York, 1951.

[104] M. M. Kostreva, Elasto-hydrodynamic lubrication: A non-linear complementarity problem, International J. Numer. Methods Fluids, 4 (1984), pp. 377-397.

[105] M. M. Kostreva, Recent results on complementarity models for engineering and economics, Inform. J. Canadian OR Society, 28 (1990), pp. 324-334.

[106] M. KoČVARA And J. V. OUtrata, A nonsmooth approach to optimization problems with equilibrium constraints, in [47], pp. 148-164.

[107] M. KoČVARA AND J. V. OUtrata, On the solution of optimum design problems with variational inequalities, in Recent Advances in Nonsmooth Optimization, World Scientific Publishers, Singapore, 1995, pp. 172-192.

[108] B. M. KWAK, Complementarity problem formulation of three-dimensional frictional contact, J. Appl. Mech., 58 (1991), pp. 134-140.

[109] B. M. KWAK AND B. C. LEE, A complementarity problem formulation for two-dimensional frictional contact problem, Comput. \& Structures, 18 (1988), pp. 469-480.

[110] L. J. LeBlanc, E. K. Morlok, and W. P. Pierskalla, An efficient approach to solving the road network equilibrium traffic assignment problem, Transportation Research, 9 (1975), pp. 309-318.

[111] S. S. LEE, A computational method for frictional contact problem using finite element method, Internat. J. Numer. Methods Engrg., 37 (1994), pp. 217-228.

[112] C. E. Lemke, Bimatrix equilibrium points and mathematical programming, Management Science, 11 (1965), pp. 681-689.

[113] C. E. Lemke And J. T. Howson, Equilibrium points of bimatrix games, SIAM J. Appl. Math., 12 (1964), pp. 413-423.

[114] F. Lopez-de-Silanes, J. R. Markusen, and T. F. Rutherford, Complementarity and increasing returns in intermediate inputs, Journal of Development Economics, 45 (1994), pp. $133-151$.

[115] P. LötstedT, Coulomb friction in two-dimensional rigid body systems, Zeitschrift für Angewandte Mathematik und Mechanik, 61 (1981), pp. 605-615.

[116] P. Lötstedt, Mechanical systems of rigid bodies subject to unilateral constraints, SIAM J. Appl. Math., 42 (1982), pp. 281-296.

[117] Z.-Q. LuO, J. S. PANG, AND D. RAlPh, Mathematical Programs with Equilibrium Constraints, Cambridge University Press, Cambridge, 1996.

[118] T. L. Magnanti, Models and algorithms for predicting urban traffic equilibria, in Transportation Planning Models, M. Florian, ed., North-Holland, Amsterdam, 1984, pp. 153-186.

[119] G. MAIER, A quadratic programming approach for certain classes of nonlinear structural problems, Meccanica, 3 (1968), pp. 121-130.

[120] G. MAIER, A matrix structural theory of piecewise linear elastoplasticity with interacting yield planes, Meccanica, 5 (1970), pp. 54-66.

[121] G. MAIER, Incremental plastic analysis in the presence of large displacement and physical instabilizing effects, Internat. J. Solids Structures, 7 (1971), pp. 345-372.

[122] G. Maier And G. NOVATI, A shakedown and bounding theory allowing for nonlinear hardening and second order geometric effects with reference to discrete structural models, in Inelastic Solids and Structures, A. Sawczuk memorial volume, Pineridge Press, Swansea, 1990, pp. 451-471.

[123] O. L. Mangasarian, Equivalence of the complementarity problem to a system of nonlinear equations, SIAM J. Appl. Math., 31 (1976), pp. 89-92.

[124] O. L. MAngasarian, Generalized linear complementarity problems as linear programs, Methods Oper. Res., 31 (1979), pp. 393-402.

[125] O. L. MANGASARIAN AND M. V. SOlOdOv, Nonlinear complementarity as unconstrained and constrained minimization, Math. Programming, 62 (1993), pp. 277-298.

[126] A. S. Manne And T. F. Rutherford, International trade in oil, gas and carbon emission rights: An intertemporal general equilibrium model, The Energy Journal, 14 (1993), pp. $1-20$.

[127] P. MARCotTe, Network design problem with congestion effects: A case of bilevel programming, Math. Programming, 34 (1986), pp. 142-162.

[128] J. Markusen and R. Wigle, Nash equilibrium tariffs for the United States and Canada: The roles of country size, scale economies and capital mobility, J. Political Mobility, 97 (1989), pp. 368-386. 
[129] L. MAthiESEn, An algorithm based on a sequence of linear complementarity problems applied to a Walrasian equilibrium model: An example, Math. Programming, 37 (1987), pp. 1-18.

[130] E. Miersemann and H. D. Mittelmann, Continuation for parametrized nonlinear variational inequalities, J. Comput. Appl. Math., 26 (1989), pp. 23-34.

[131] R. D. C. Monteiro, J. S. Pang, And T. WAng, A positive algorithm for the nonlinear complementarity problem, SIAM J. Optim., 5 (1995), pp. 129-148.

[132] J. J. Moré, Global methods for nonlinear complementarity problems, Math. Oper. Res., 21 (1996), pp. 589-614.

[133] J. J. MORÉ AND G. TORALDO, On the solution of large quadratic programming problems with bound constraints, SIAM J. Optim., 1 (1991), pp. 93-113.

[134] F. H. Murphy, H. D. Sherali, And A. L. Soyster, A mathematical programming approach for determining oligopolistic market equilibrium, Math. Programming, 24 (1982), pp. 92-106.

[135] K. G. Murty, Linear Complementarity, Linear and Nonlinear Programming, HeldermanVerlag, Berlin, 1988.

[136] J. F. NASH, Equilibrium points in N-person games, Proceedings of the National Academy of Sciences, 36 (1950), pp. 48-49.

[137] K. P. Он, Analysis of a needle bearing, Trans. ASME, J. Tribology, 106 (1984), pp. 78-87.

[138] K. P. Он, The numerical solution of dynamically loaded elastohydrodynamic contact as a nonlinear complementarity problem, Trans. ASME, J. Tribology, 106 (1984), pp. 88-95.

[139] K. P. Он, The formulation of the mixed lubrication problem as a generalized nonlinear complementarity problem, Trans. ASME, J. Tribology, 108 (1986), pp. 598-604.

[140] K. P. OH AND P. K. GoenkA, The elastohydrodynamic solution of journal bearings under dynamic loading, Trans. ASME, J. Tribology, 107 (1985), pp. 389-395.

[141] K. P. OH, C. H. LI, AND P. K. GoEnKa, Elastohydrodynamic lubrication of piston skirts, Trans. ASME, J. Tribology, 109 (1987), pp. 356-362.

[142] J. V. Outrata, On the numerical solution of a class of stackelberg problems, Z. Oper. Res., 4 (1990), pp. 255-278.

[143] J. V. Outrata, On necessary optimality conditions for stackelberg problems, J. Optim. Theory Appl., 76 (1993), pp. 305-320.

[144] P. D. Panagiotopoulos, Inequality Problems in Mechanics and Applications, Birkhäuser, Boston, 1985

[145] P. D. Panagiotopoulos, Hemivariational Inequalities, Springer-Verlag, Berlin, 1993.

[146] P. D. Panagiotopoulos And A. M. Al-FAhed, Robot hand grasping and related problems: Optimal control and identification, Internat. J. Robotics Research, 13 (1994), pp. 127-136.

[147] J. S. PANG, A B-differentiable equation based, globally and locally quadratically convergent algorithm for nonlinear programs, complementarity and variational inequality problems, Math. Programming, 51 (1991), pp. 101-132.

[148] J. S. PANG, Complementarity problems, in Handbook in Global Optimization, R. Horst and P. Pardalos, eds., Kluwer Academic Publishers, Boston, 1994.

[149] J. S. PANG AND S. A. GABRIEL, NE/SQP: A robust algorithm for the nonlinear complementarity problem, Math. Programming, 60 (1993), pp. 295-338.

[150] J. S. PANG AND L. QI, Nonsmooth equations: Motivation and algorithms, SIAM J. Optim., 3 (1993), pp. 443-465.

[151] J. S. PANG AND J. C. TRINKLE, Complementarity formulations and existence of solutions of multi-rigid-body contact problems with Coulomb friction, Math. Programming, 73 (1996), pp. 199-226.

[152] J. C. Trinkle And J. S. PAng, Dynamic multi-rigid-body systems with concurrent distributed contacts friction, IEEE International Conference on Robotics and Automation, 1997, pp. 2276-2281.

[153] J. S. PAng, J. C. Trinkle, And G. LO, A complementarity approach to a quasistatic multirigid-body contact problem, Computat. Optim. Appl., 5 (1996), pp. 97-138.

[154] J. S. PANG AND C. S. YU, Linearized simplicial decomposition methods for computing traffic equilibria on networks, Networks, 14 (1984), pp. 427-438.

155] C. Perroni And T. Rutherford, International trade in carbon emission rights and basic materials: General equilibrium calculations for 2020, Scandinavian J. Economics, (1993).

[156] C. Perroni And T. Rutherford, Regular flexibility of nested CES functions, European Economic Review, 39 (1995), pp. 335-343.

[157] J. Petersson, Optimization of Structures in Unilateral Contact, Linköping studies in science and technology, dissertations, Division of Mechanics, Department of Mechanical Engineering, Linköping University, Linköping, Sweden, 1995.

[158] P. Petri, Modelling Japanese-American Trade, A Study of Asymmetric Interdependence, Harvard University Press, Cambridge, MA, 1984. 
[159] F. Pfeiffer And C. Glocker, Multibody Dynamics with Unilateral Contact, John Wiley \& Sons, New York, 1996.

[160] L. QI AND X. Chen, A Globally Convergent Successive Approximation Method for Nonsmooth Equations, Tech. rep., School of Mathematics, University of New South Wales, Sydney, 1993.

[161] V. T. Rajan, R. Burridge, And J. T. Schwartz, Dynamics of a rigid body in frictional contact with rigid walls, IEEE International Conference on Robotics and Automation, Raleigh, NC, 1987, pp. 671-677.

[162] D. RALPH, Global convergence of damped Newton's method for nonsmooth equations, via the path search, Math. Oper. Res., 19 (1994), pp. 352-389.

[163] S. M. Robinson, Newton's method for a class of nonsmooth functions, Set Valued Analysis, 2 (1994), pp. 291-305.

[164] R. T. Rockafellar, Convex Analysis, Princeton University Press, Princeton, NJ, 1970.

[165] R. T. RockAfellaR, Linear-quadratic programming and optimal control, SIAM J. Control Optim., 25 (1987), pp. 781-814.

[166] R. T. RockAfellaR, Computational schemes for large-scale problems in extended linearquadratic programming, Math. Programming, 48 (1990), pp. 447-474.

[167] R. T. RockAfellar AND R. J.-B. Wets, A Lagrangian finite generation technique for solving linear-quadratic problems in stochastic programming, Math. Programming Study, 28 (1986), pp. 63-93.

[168] R. T. RockAfellar AND R. J.-B. Wets, Linear-quadratic programming problems with stochastic penalties: The finite generation algorithm, in Stochastic Optimization, V. I. Arkin, A. Shiraer, and R. J.-B. Wets, eds., Lecture Notes in Control and Information Sciences, IIASA Series No. 81, Springer-Verlag, New York, Berlin, 1987, pp. 545-560.

[169] R. T. RockAfellar AND R. J.-B. Wets, Generalized linear-quadratic problems of deterministic and stochastic optimal control in discrete time, SIAM J. Control Optim., 28 (1990), pp. 810-822.

[170] H. C. Rodrigues, A mixed variational formulation for shape optimization of solids with contact conditions, Structural Optimization, 6 (1993), pp. 19-28.

[171] J. F. Rodrigues, Obstacle Problems in Mathematical Physics, Elsevier Publishing Company, Amsterdam, 1987.

[172] T. F. Rutherford, A Modeling System for Applied General Equilibrium Analysis, Cowles Foundation Discussion Paper 836, Yale University, New Haven, CT, May 1987.

[173] T. F. Rutherford, MPSGE, Tech. rep., University of Western Ontario, London, Ontario, 1988.

[174] T. F. Rutherford, Applied general equilibrium modeling with MPSGE as a GAMS subsystem, Department of Economics, University of Colorado, Boulder, Jan. 1994, manuscript.

[175] T. F. RuthERFORD, Extensions of GAMS for complementarity problems arising in applied economic analysis, J. Econom. Dynam. Control, (1995).

[176] H. E. Scarf, The Computation of Economic Equilibria, Yale University Press, New Haven, CT, 1973.

[177] W. Seyfferth And F. Pfeiffer, Dynamics of assembly processes with a manipulator, Proceedings of the 1992 IEEE/RSJ International Conference on Intelligent Robots and Systems, 1992, pp. 1303-1310.

[178] H. D. Sherali, A. L. Soyster, And F. H. Murphy, Stackelberg-Nash-Cournot equilibria: Characterization and computation, Oper. Res., 31 (1983), pp. 253-276.

[179] J. Shoven And J. Whalley, Applied general equilibrium models of taxation and international trade: Introduction and survey, J. Economic Literature, 22 (1984), pp. 1007-1051.

[180] E. Simantiraki And D. F. Shanno, An Infeasible-Interior-Point Algorithm for Solving Mixed Complementarity Problems, Research Report 37-95, Rutgers Center for Operations Research, Rutgers University, New Brunswick, NJ, 1995.

[181] M. J. Sмiтh, The existence, uniqueness and stability of traffic equilibria, Transportation Research, 13B (1979), pp. 295-304.

[182] M. V. Solodov And P. TSEng, Modified projection-type methods for monotone variational inequalities, SIAM J. Control Optim., 34 (1996), pp. 1814-1830.

[183] T. Srinivasan and J. Whalley, General Equilibrium Trade Policy Modelling, MIT Press, Boston, 1986.

[184] H. V. Stackelberg, The Theory of Market Economy, Oxford University Press, London, 1952.

[185] G. E. Stavroulakis, Optimal prestress of cracked unilateral structures: Finite element analysis of an optimal control problem for variational inequalities, Comput. Methods Appl. Mech. Engrg., (1996). 
[186] G. E. Stavroulakis, Optimal prestress of structures with frictional unilateral contact interfaces, Arch. Appl. Mech., (1996).

[187] G. E. Stavroulakis, P. D. Panagiotopoulos, and A. M. Al-Fahed, On the rigid body displacements and rotations in unilateral contact problems and applications, Comput. \& Structures, 40 (1991), pp. 599-614.

[188] P. A. Steenbrink, Optimization of Transport Networks, John Wiley \& Sons, London, 1974.

[189] D. E. Stewart And J. C. Trinkle, An implicit time-stepping scheme for rigid body dynamics with inelastic collisions and Coulomb friction, Internat. J. Numer. Methods Engrg., 39 (1996), pp. 2673-2691.

[190] D. C. Sun, A thermal elastic theory of piston-ring and cylinder-bore contact, J. Appl. Mech., 58 (1991), pp. 141-153.

[191] S. M. Sun, M. C. NATORI, AND K. C. PARK, A computational procedure for flexible beams with frictional contact constraints, Internat. J. Numer. Methods Engrg., 36 (1993), pp. 37813800.

[192] F. Tin-LOI AND J. S. MisA, Large displacement elastoplastic analysis of semirigid steel frames, Internat. J. Numer. Methods Engrg., 39 (1996), pp. 741-762.

[193] F. TIN-LOI AND J. S. PANG, Elastoplastic analysis of structures with nonlinear hardening, Comput. Methods Appl. Mech. Engrg., 107 (1993), pp. 299-312.

[194] F. Tin-Loi And V. Vimonsatit, Nonlinear Analysis of Semi-Rigid Frames: A Parametric Complementarity Approach, Engrg. Structures, 18 (1996), pp. 115-124.

[195] R. L. ToBin, A variable dimension solution approach for the general spatial equilibrium problem, Math. Programming, 40 (1988), pp. 33-51.

[196] R. L. ToBin, Uniqueness results and algorithm for Stackelberg-Cournot-Nash equilibria, Ann. Oper. Res., 34 (1992), pp. 21-36.

[197] M. J. TodD, Computation of Fixed Points and Applications, Lecture Notes in Econom. and Math. Systems 124, Springer-Verlag, Heidelberg, 1976.

[198] J. C. Trinkle, J. S. Pang, S. Sudarsky, And G. LO, On dynamic multi-rigid-body contact problems, Z. Angew. Math. Mech., 77 (1997), pp. 267-279.

[199] P. Tseng, N. Yamashita, And M. Fukushima, Equivalence of complementarity problems to differentiable minimization: A unified approach, SIAM J. Optim., 6 (1996), pp. 446-460.

[200] H. R. VARIAN, Microeconomic Analysis, W.W. Norton \& Company, New York, 1978.

[201] R. R. WAKEFIELD AND F. TIN-LOI, Large scale nonholonomic elastoplastic analysis using a linear complementarity formulation, Comput. Methods Appl. Mech. Engrg., 84 (1990), pp. 229-242.

[202] R. R. WAKEFiELd AND F. TiN-Loi, Large displacement elastoplastic analysis of frames using an iterative LCP approach, Internat. J. Mech. Sci., 33 (1991), pp. 379-391.

[203] L. Walras, Elements of Pure Economics, Allen and Unwin, London, 1954.

[204] T. Wang, R. D. C. Monteiro, And J. S. Pang, An interior point potential reduction method for constrained equations, Math. Programming, 74 (1996), pp. 159-196.

[205] J. G. WARdrop, Some theoretical aspects of road traffic research, Proceeding of the Institute of Civil Engineers, Part II, 1952, pp. 325-378.

[206] P. Wilmott, J. Dewynne, And S. Howison, Option Pricing, Oxford Financial Press, Oxford, 1993.

[207] S. J. WRIGHT AND D. RALPh, A superlinear infeasible-interior-point algorithm for monotone complementarity problems, Math. Oper. Res., 21 (1996), pp. 815-838.

[208] B. Xiao AND P. T. HARker, A nonsmooth Newton method for variational inequalities: II: Numerical results, Math. Programming, 65 (1994), pp. 195-216.

[209] S. T. Yau And Y. GaO, Obstacle problem for von Kármán equations, Adv. Appl. Math., 13 (1992), pp. 123-141.

[210] C. Y. ZHU, Modified proximal point algorithm for extended linear-quadratic programming, Comput. Optim. Appl., 2 (1992), pp. 182-205.

[211] C. Y. Zhu And R. T. RockAfellar, Primal-dual projected gradient algorithms for extended linear-quadratic programming, SIAM J. Optim., 3 (1993), pp. 751-783. 